\title{
DNA Methylation Reduces the Yes-Associated Protein 1/WW Domain Containing Transcription Regulator 1 Pathway and Prevents Pathologic Remodeling during Bladder Obstruction by Limiting Expression of BDNF
}

\author{
Martin Sidler, ${ }^{* \dagger}$ Karen J. Aitken, ${ }^{* \dagger}$ Jia-Xin Jiang, ${ }^{* \S}$ Chris Sotiropoulos, ${ }^{\star \S}$ Prateek Aggarwal, ${ }^{* \llbracket}$ Arsalan Anees, \\ Cynthia Chong, ${ }^{,}$Aliza Siebenaller, ${ }^{*}$ Thenuka Thanabalasingam, ${ }^{*}$ J J. Maxwell White, ${ }^{*}$ Sanaa Choufani, ${ }^{* *}$

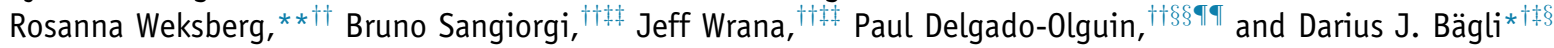

From Developmental and Stem Cell Biology,* Genetics and Genome Biology, ** and Translational Medicine, ${ }^{\S \S}$ the Research Institute, and the Urology Division, ${ }^{\dagger}$ Department of Surgery, The Hospital for Sick Children, Toronto, Ontario; the Institute of Medical Sciences, ${ }^{\ddagger}$ Faculty of Medicine, the Department of Physiology, ${ }^{\S}$ Faculty of Arts and Sciences, the Human Biology Program, ${ }^{\top}$ and the Department of Molecular Genetics, ${ }^{\dagger \dagger}$ University of Toronto, Toronto, Ontario; the School of Business, "Queen's University, Kingston, Ontario; the Lunenfeld-Tanenbaum Research Institute, ${ }^{\ddagger \ddagger}$ Mount Sinai Hospital, Joseph and Wolf Lebovic Health Complex, Toronto, Ontario; and the Heart and Stroke/Richard Leward Centre of Excellence in Cardiovascular Research, ${ }^{\top \uparrow}$ Toronto, Ontario, Canada

\author{
Accepted for publication \\ June 26, 2018. \\ Address correspondence to \\ Darius J. Bägli, M.D., The \\ Hospital for Sick Children, 555 \\ University Ave., M299, \\ Toronto, ON M5G1X8, \\ Canada. E-mail: darius.bagli@ \\ sickkids.ca.
}

\begin{abstract}
Chronic bladder obstruction and bladder smooth muscle cell (SMC) stretch provide fibrotic and mechanical environments that can lead to epigenetic change. Therefore, we examined the role of DNA methylation in bladder pathology and transcriptional control. Sprague-Dawley female rats underwent partial bladder obstruction by ligation of a silk suture around the proximal urethra next to a $0.9-\mathrm{mm}$ steel rod. Sham operation comprised passing the suture around the urethra. After 2 weeks, rats were randomized to normal saline or DNA methyltransferase inhibitor, 5-aza-2-deoxycytidine (DAC) at $1 \mathrm{mg} / \mathrm{kg}$, three times/week intraperitoneally. After 6 weeks, bladders were weighed and divided for histology and RNA analysis by high-throughput real-time quantitative PCR arrays. DAC treatment during obstruction in vivo profoundly augmented brain-derived neurotrophic factor (BDNF) expression compared with the obstruction with vehicle group, which was statistically correlated with pathophysiologic parameters. BDNF, cysteine rich angiogenic inducer 61 (CYR61), and connective tissue growth factor (CTGF) expression clustered tightly together using Pearson's correlation analysis. Their promoters were associated with the TEA domain family member 1 (TEAD1) and Yes-associated protein $1 /$ WW domain containing transcription regulator 1 pathways. Interestingly, DAC treatment increased BDNF expression in bladder SMCS $(P<0.0002)$. Stretch-induced BDNF was inhibited by the YAP/WWTR1 inhibitor verteporfin. Verteporfin improved the SMC phenotype (proliferative markers and SMC marker expression), in part by reducing BDNF. Expression of BDNF is limited by DNA methylation and associated with pathophysiologic changes during partial bladder outlet obstruction and SMC phenotypic change in vitro. (Am J Pathol 2018, 188: 2177-2194; https://doi.org/10.1016/j.ajpath.2018.06.024)
\end{abstract}

Partial bladder outlet obstruction (PBO), occurring in diseases such as prostate hyperplasia and urethral valves, is a widespread cause of urinary dysfunction, patient discomfort, and overactive bladder. It was responsible for $\$ 65.9$ billion in US 2007 health care costs. ${ }^{1}$ Although removing the obstruction partially improves the functional and pathologic sequelae, ongoing symptoms remain in
Supported by the Canadian Institutes of Health Research (D.J.B., P.D.O.), the Strategic Training program in regenerative medicine (TPRM) (K.J.A.), the Research Training competition, SickKids Hospital Research Institute (J.-X.J.), the Department of Physiology at the University of Toronto (J.-X.J.), the Heart and Stroke Foundation of Canada (P.D.-O.), YMCA Canada (A.S.), and the Natural Sciences and Engineering Research Council of Canada (P.D.-O.).

M.S. and K.J.A. contributed equally to this work.

Disclosures: None declared. 
many patients. Epigenetic changes, such as DNA methylation, play an important role in both adaptive and recovery processes, including fibrosis and proliferation. ${ }^{2,3}$ Epigenetic mechanisms may be critical components of the response to obstructive stimuli (matrix, hypoxia, inflammatory, and mechanical stimuli). Because DNA methylation may be crucial to the maladaptive or adaptive responses to these stimuli, a hypomethylating agent was used in a rodent model of PBO to evaluate the role of DNA methylation in bladder remodeling and gene expression during obstruction. This invites investigation of potential epigenetic mechanisms in bladder tissue homeostasis and repair.

During aging, global loss of DNA methylation occurs across the genome. The aging bladder is commonly clinically associated with dysfunctional changes that include the gradual onset of irritative and obstructive bladder symptoms. However, it is not known if DNA methylation may also contribute to the clinical pathology of the aging bladder. In vitro, DNA methylation changes dynamically in response to obstructive stimuli in bladder smooth muscle cells alongside proliferation. ${ }^{3}$ In this study, we investigated the effect of DNA methylation during obstruction of nonaging rats to isolate DNA methylation from other age-related pathologies. Functional bladder parameters were assessed using metabolic cages and associated gene expression during obstruction in nonaging rats while inhibiting DNA methylation. By studying candidate gene expression, the most profound and significant changes in gene expression among all groups were identified as brainderived neurotrophic factor (BDNF) and connective tissue growth factor (CTGF). Using correlation analysis, novel and discrete changes in gene expression in Bdnf and CTGF, relevant to obstructive bladder dysfunction, were identified. It was further determined that BDNF, cysteine rich angiogenic inducer 61 (CYR61), and CTGF share transcriptional regulation through the YAP/WWTR1 pathway. BDNF's massive increase in transcript level and localization around smooth muscle bundles led us to investigate its role more clearly. BDNF can induce highly contrasting effects during bladder obstruction, ${ }^{4,5}$ including overactivity, but also restoration of neuronal function. The functional role of BDNF on bladder smooth muscle phenotype has previously not been examined in detail, despite potential implications for regulation of contractile machinery. We examined the role of DNA methylation and YAP/WWTR1 in gene expression and their coordinate effects on bladder smooth muscle cell (BSMC) pathophysiology.

\section{Materials and Methods}

\section{Partial Bladder Outlet Obstruction}

Partial bladder outlet obstruction was performed as described, ${ }^{6}$ under a protocol approved by the Animal Care Committee at The Hospital for Sick Children Research Institute, following policies established in the Canadian Council on Animal Care Guide to the Care and Use of Experimental Animals. In this design, 200 to $250 \mathrm{~g}$ Sprague-Dawley female rats $(n=20$; Charles River Laboratories, Wilmington, MA) underwent PBO by tying a silk suture around the proximal urethra and a $0.9-\mathrm{mm}$ steel rod; the rod was then removed, leaving the suture behind to form the partial obstruction. Sham operation was performed without tying the suture $(n=12) .{ }^{6}$ Pain medication was given for the first 24 hours and whenever symptoms of pain were evident.

\section{Decitabine Treatment}

Hypomethylating activity of 5-aza-2'-deoxycytidine (or decitabine; DAC; LC Laboratories, Woburn, MA) treatment was tested by examining Line-1 methylation in the spleens of rats during a separate dose-finding experiment (Supplemental Figure S1). After 2 weeks of obstruction, half of each group was treated with the DNA methyltransferase inhibitor, DAC, at $1 \mathrm{mg} / \mathrm{kg}$, three times/week intraperitoneally for 4 weeks (Supplemental Figure S2). Control animals received normal saline in the same course and route as the treatment. ${ }^{6}$

\section{Micturition Measurements}

Micturition patterns were recorded at 6 weeks 1 to 2 days before sacrifice, using previously described methods. ${ }^{7-9}$ Voiding patterns and volumes (Supplemental Figure S3) were recorded on a computer using Logger Pro software version 3.9 (Vernier Software \& Technology, Beaverton, OR). Residual volumes were measured in anesthetized rats at the time of sacrifice. ${ }^{6}$

\section{SMC Culture and Mechanical Stretch}

Human BSMCs (Sciencell, Carlsbad, CA) were maintained in BSMC media including growth supplements, fetal bovine serum, and antibiotic/antimycotic (Sciencell). Cells were plated onto collagen I-coated BioFlex plates (Flexcell International, Burlington, NC) at $4 \times 10^{4} \mathrm{cells} / \mathrm{mL}$ in a 1:1 mix of BSMC medium plus Eagle's minimal essential medium (EMEM) (Wisent, St. Bruno, QC, Canada) plus antibiotic/antimycotic (Wisent). After 24 to 48 hours, cells were serum starved for 1 day in EMEM, then treated in EMEM plus either vehicle (dimethyl sulfoxide), verteporfin (VP; $0.1 \mu \mathrm{mol} / \mathrm{L}$; MilliporeSigma, Burlington, MA), or decitabine (DAC; $2 \mu \mathrm{mol} / \mathrm{L}$ ), 1 hour. Cells were mechanically strained to 5\% static elongation on the Flexcell 3000 (Flexcell International) for 6, 8, or 24 hours, using a ramping pattern, as previously described. ${ }^{10,11}$ For exogenous BDNF, Trk inhibitor GNF 5837 (both from Alamone Labs, Jerusalem, Israel), and verteporfin treatments, human BSMCs were plated on denatured collagen-coated glass coverslips, ${ }^{12}$ and serum starved overnight before treatments. BDNF was added to serum-starved cells at $0,2.5,5$, 
or $10 \mathrm{ng} / \mathrm{mL}$ in serum-free EMEM. VP or vehicle (dimethyl sulfoxide at same volume) was added to BSMCs. An initial dosage finding study on human BSMCs showed that VP was not cytotoxic at this level, because it did not decrease cell counts or morphology in unstretched cells (data not shown). Neonatal Sprague-Dawley rat bladder domes were minced and digested using collagenase type IV (Elastin Products Company, Owensville, MO). Digests were washed and cells were preplated to remove fibroblasts. Passaging cells with $0.25 \mathrm{mmol} / \mathrm{L}$ trypsin removed contaminating urothelial cells. Cells were plated at passage 3, as late passage primary rat SMC loose differentiation markers. Rat neonatal SMCs were serum starved and stretched in a static ramping pattern for 8 hours, as previously described. ${ }^{10}$

\section{Collagen Gel Contraction Assay}

Collagen gels were assembled using neutralized type I collagen $(2.4 \mathrm{mg} / \mathrm{mL}$; Elastin Products Company, Owensville, MO) and plating into 24-well plates. Serum-starved cells that had been pretreated with vehicle or $10 \mathrm{ng} / \mathrm{mL}$ BDNF for 2 days were plated at $5 \times 10^{3}$ cells $/ \mathrm{mL}$ onto collagen gels in EMEM. After 2 hours to allow for cell attachment, cells were then treated with vehicle, $10 \mathrm{ng} / \mathrm{mL}$ BDNF, or BDNF + GNF $5837(10 \mu \mathrm{mol} / \mathrm{L})$, then gels were released from the sidewall of the 24-well plate for another 2 , 4 , and 18 hours.

\section{RNA Isolation, Custom HT-qPCR Array, and Quantitative RT-PCR}

Bladder tissues were harvested, treated in RNAlater at $4^{\circ} \mathrm{C}$ for 48 hours, dried on sterile gauze, and stored at $-80^{\circ} \mathrm{C}$ until RNA isolation. Tissue samples were crushed under liquid nitrogen, then homogenized at $4^{\circ} \mathrm{C}$ in Trizol (Thermo Fisher, Waltham, MA) using a refrigerated Bullet Blender (Next Advance, Troy, NY) with steel 0.3 to $1.5 \mathrm{mmol} / \mathrm{L}$ pellets for 3 minutes at setting 8 . After the steel pellets settled by gravity, the supernatant was processed for RNA isolation. ${ }^{6}$ Samples from cell culture experiments were isolated by adding Trizol to wells and proceeding as previously described. ${ }^{11}$ Each RNA sample from groups $\mathrm{PBO}, \mathrm{PBO}+\mathrm{DAC}$, and sham $(n=4)$ was reverse transcribed using Quant-it (Qiagen, Hilden, Germany) and assessed by high-throughput real-time quantitative PCR (HT-qPCR) with a custom array using built-in quality checks. The 85 candidate genes were selected through in silico curation of literature on bladder obstruction and bladder smooth muscle dedifferentiation (Supplemental Table S1). The HTqPCR package on $\mathrm{R}$ was used to identify statistical significance of the custom array by limma and $t$-test. ${ }^{13}$ Five reference genes, Rpll3a, B2M, Hprt1, Ldha, and Rplp1, plus $\beta$-actin were assessed for optimal normalization on Excel (Microsoft Corp., Redmond, WA) and then the $\Delta \Delta \mathrm{Ct}$ method. Confirmation of significant HT-qPCR results was performed on a larger set of RNA samples by real-time quantitative PCR of cDNA produced by reverse transcription with Superscript III and Oligo d(t) (Thermo Fisher) and PCR amplification with iQ SYBR Green Supermix (BioRad, Hercules, CA). ${ }^{3,14}$ Primers are defined in Table 1. Total Ntrk2 expression of neonatal rat BSMC obstructed and sham bladders was assayed using rat Ntrk2 primers from Qiagen. Analysis of variance and post hoc $t$-tests were performed to identify significant changes in gene expression.

\section{Transcription Factor Binding Site Analysis and Pathway Analysis}

To predict transcription factors and pathways of importance for the clustered genes of BDNF, CYR61, and CTGF, the promoters of these genes were analyzed using the webbased programs, TOPGO and OPOSSUM 3.0 (http:// opossum.cisreg.ca, last accessed October 31, 2017).

\section{Western Blotting of BSMCs}

Protein extracts from mechanical strain and in vivo obstruction experiments were isolated in radioimmunoprecipitation assay buffer plus protease inhibitor cocktail (Roche, Basel, Switzerland), at $4^{\circ} \mathrm{C}$. They were quantitated using the Pierce Bradford Protein quantification kit (Thermo Fisher). Protein samples $(10 \mu \mathrm{g})$ were electrophoresed on $10 \%$ or gradient polyacrylamide gels (BioRad) and transferred to nitrocellulose membranes (Amersham, GE Healthcare, Little Chalfont, UK). ${ }^{11}$ Membranes were blocked in skim milk and bovine serum albumin, then probed using antibodies against smooth muscle myosin (rabbit; MilliporeSigma), calponin (mouse; MilliporeSigma), and reference proteins total actin (rabbit; MilliporeSigma) and glyceraldehyde-3-phosphate dehydrogenase (rabbit; Cell Signaling Technology, part of NEB, Ipswich, MA), with secondary anti-rabbit or anti-mouse horseradish peroxidase conjugates (Cell Signaling Technology), as appropriate. Autoradiographs were developed using electrochemiluminescence (Amersham/GE Healthcare). Normalization and quantification were performed on ImageJ version $1.51(\mathrm{NIH}$, Bethesda, MD; http://imagej. nih.gov/ij) by analysis of band densities from equivalent band areas on blots for reference proteins compared with target proteins.

\section{Enzyme-Linked Immunosorbent Assay for Tissue BDNF}

Bladder tissues were washed in phosphate-buffered saline (PBS) and frozen as for RNA isolation. Protein was isolated and quantitated as for Western blotting in radioimmunoprecipitation assay buffer. Protein lysates $(10 \mu \mathrm{g})$ and standards were added to the Chemikine BDNF sandwich enzyme-linked immunosorbent assay kit immunoassay plates, following the manufacturer's instructions (R\&D Systems, Minneapolis, MN). 
Table 1 qPCR Oligonucleotide Sequences

\begin{tabular}{|c|c|c|c|}
\hline Target & Forward & Reverse & $\begin{array}{l}\text { Amplicon } \\
\text { size, bp }\end{array}$ \\
\hline $\begin{array}{l}\text { Human BDNF promoter } \\
\text { gDNA (ChIP primer in } \\
\text { gene desert) }\end{array}$ & 5'-AGCCCAACAACTTTCCCTTT-3' & 5'-CGGGGCTGTTAACTCACATT-3' & 162 \\
\hline $\begin{array}{l}\text { Human BDNF promoter } \\
\text { gDNA (ChIP primer) }\end{array}$ & $5^{\prime}-\mathrm{AGATCACAGAGCCTGCCAGT-3^{ \prime }}$ & 5'-AATTTGTGTGAGGGGTCTCG-3' & 177 \\
\hline $\begin{array}{l}\text { Human BDNF promoter } \\
\text { gDNA at EGR1 site } \\
\text { (ChIP primer) }\end{array}$ & 5'-AGCCTTTCGGGTTCTCATTT-3' & 5'-AGGTCCAGGGACTCCAAGTT-3' & 196 \\
\hline Human BDNF cDNA & $5^{\prime}-$ TAACGGCGGCAGACAAAAAGA - 3' & 5'-GAAGTATTGCTTCAGTTGGCCT-3' & 101 \\
\hline Human and rat CTGF cDNA & $5^{\prime}-$ AAGACCTGTGGGATGGGC- $3^{\prime}$ & $5^{\prime}-$ TGGTGCAGCCAGAAAGCTC-3' & 194 \\
\hline Human CYR61 cDNA & $5^{\prime}$-CGAGGTGGAGTTGACGAGAAA-3' & $5^{\prime}$-CTTTGAGCACTGGGACCATGA-3' & 159 \\
\hline Human YAP cDNA & 5'-ACCCTCGTTTTGCCATGAAC-3' & 5'-TGTGCTGGGATTGATATTCCGTA-3' & 221 \\
\hline Human TAZ/WWTR1 cDNA & $5^{\prime}$-CCATCACTAATAATAGCTCAGATC-3' & $5^{\prime}$-GTGATTACAGCCAGGTTAGAAAG-3' & 338 \\
\hline Human $\beta$-actin cDNA & $5^{\prime}-\mathrm{CGGCTACAGCTTCACCACCA-3^{ \prime }}$ & $5^{\prime}$-CGGGCAGCTCGTAGCTCTTC-3' & 140 \\
\hline Human B2M cDNA & $5^{\prime}$-AGGCTATCCAGCGTACTCCA-3' & $5^{\prime}-$ TCATCCAATCCAAATGCGGC - $3^{\prime}$ & 348 \\
\hline Human GAPDH cDNA & $5^{\prime}$-GTCAGTGGTGGACCTGACCT-3' & 5'-TGCTGTAGCCAAATTCGTTG-3' & 245 \\
\hline Human HPRT cDNA & 5'-GGCGAACCTCTCGGCTTT-3' & 5'-CATCACTAATCACGACGCCA-3' & 159 \\
\hline Human SDHA cDNA & $5^{\prime}$-ACTGTTGCAGCACAGCTAGAA-3' & $5^{\prime}-$ TTATGCGATGGATGGACCG-3' & 283 \\
\hline Human UBC cDNA & 5'-TTAGGACGGGACTTGGGTGA-3' & 5'-TCACGAAGATCTGCATTGTCAAG-3' & 240 \\
\hline Rat total (pan) Bdnf cDNA & 5'-TGGGGAGCTGAGCGTGTGTGA-3' & 5'-TGTGACCGTCCCGCCAGACAT-3' & 90 \\
\hline Rat CYR61 cDNA & 5'-CTTCCTGTCTTTGGCACGGA-3' & $5^{\prime}$-AACTCGTGTGGAGATGCCAG- $3^{\prime}$ & 134 \\
\hline Rat Tead1 cDNA & 5'-TTTGTGCAGCAGGCCTACCCCATC-3' & 5'-GGCGAAGCTTGGTTGTGCCAATGGA-3' & 129 \\
\hline Rat Tead4 cDNA & 5'-ACAGTGACCCCTACCTCGAA-3' & 5'-GGTCTGCCCAGAACTTCACA-3' & 135 \\
\hline Rat Wwtr1 cDNA & 5'-GAGGAAGGGCTCGCTTTTGT - 3' & $5^{\prime}$-ATGTTGACCTCGGGACTTTGG-3' & 88 \\
\hline Rat Yap cDNA & $5^{\prime}-$ АATATCAATCCCAGCACAGCA-3' & 5'-САTTCTGAGTCCCTCCATCC-3' & 109 \\
\hline Rat Ntrk2 cDNA (TrkB T1) & 5'-GAGCATCTCTCGGTCTATGC-3' & $5^{\prime}$-CCCATCCAGGGGGATCTTAT-3' & 146 \\
\hline Rat Ntrk2 cDNA (total) & Qiagen & Qiagen & 71 \\
\hline Rat $\beta$-actin cDNA & $5^{\prime}$-GGTCGTACCACTGGCATTGTG-3' & $5^{\prime}$-GCTCGGTCAGGATCTTCATGAG-3' & 150 \\
\hline Rat Gapdh cDNA & $5^{\prime}$-GATCGTGGAAGGGCTAATGA-3' & $5^{\prime}$-GAGCTCTGGGATGACTTTGC-3' & 165 \\
\hline Rat Hprt cDNA & $5^{\prime}$-AGGCCAGACTTTGTTGGATT-3' & 5'-GCTTTTCCACTTTCGCTGAT-3' & 118 \\
\hline Rat Rpl32 cDNA & 5'-CATCTGTTTTGCGGCATCA-3' & $5^{\prime}$-CACCCTGTTGTCGATGCCTC-3' & 152 \\
\hline
\end{tabular}

ChIP, chromatin immunoprecipitation; EGR, early growth response; gDNA, genomic DNA; qPCR, real-time quantitative PCR.

\section{Immunofluorescence}

Cells or $7 \mu \mathrm{mol} / \mathrm{L}$ equatorial bladder cryosections were fixed in paraformaldehyde and washed twice in PBS. Antigen exposure was performed by treatment with $0.2 \%$ Triton X100. Blocking was performed in normal donkey serum (Jackson ImmunoResearch Laboratories, West Grove, PA). Then, staining was performed with the smooth muscle myosin heavy chain rabbit antibody (Medical Group/Alfa Aesar, Tewksbury, MA; or MilliporeSigma), calponin (MilliporeSigma), collagen type I (MilliporeSigma), BDNF (rabbit host; Abclonal, Woburn, MA), YAP, mouse host YAP (Santa Cruz Biotechnology, Dallas, TX), rabbit host YAP/TAZ and phosphorylated YAP (Cell Signaling, a part of NEB), WWTR1 [mouse host (R\&D Systems) or rabbit host (Novus Biologicals, Littleton, CO)], and secondary donkey anti-rabbit-Farred Fab fragments or donkey antimouse-Cy3 Fab (Jackson ImmunoResearch Laboratories). Nuclei were stained with Hoechst. Slides were mounted with Dako fluorescence mounting medium (Agilent, Santa Clara,
CA). Immunofluorescence was captured using an Olympus IX81 microscope (Olympus Corp., Tokyo, Japan) equipped with a Hamamatsu camera (Hamamatsu Photonics K.K, Hamamatsu, Japan) and spectral borealis lasers attached to Volocity software version 6.2 (Perkin Elmer, Waltham, MA). To test for myocyte hypertrophy, transnuclear cross sections of myocytes ${ }^{15}$ were measured on Volocity. Intensity of immunofluorescence signals in muscle bundles was evaluated by normalizing the sum of the intensity for each smooth muscle bundle region to the number of cells in each bundle. Immunofluorescence staining for nerves was performed with the $\beta 3$ tubulin rabbit antibody (MilliporeSigma). Additional processing for both $\beta 3$ tubulin and YAP antibodies included preclearing treatment with ice-cold methanol for 10 minutes, antigen exposure with $0.3 \%$ Triton X-100, blocking in $10 \%$ bovine serum albumin (MilliporeSigma) and $10 \%$ normal donkey serum (Jackson ImmunoResearch Laboratories), for 30 minutes, and washing steps in $0.1 \%$ Triton $\mathrm{X}-100$ in PBS. 


\section{Bladder Histology and Wall Thickness}

Equatorial bladder cryosections (7 $\mu \mathrm{m}$ thick) were stained with PicroSirius Red or hematoxylin and eosin. Sections were scanned and imaged using the Aperio system to ascertain the wall thickness at equatorial regions of the bladder, and degree of collagen accumulation by in muscle and nonmuscle tissue compartments. ${ }^{6}$ Data were included in Pearson's correlations.

\section{Chromatin Immunoprecipitation PCR for YAP/WWTR1 Association with BDNF Genomic Regions during Stretch}

Chromatin was isolated from human bladder smooth muscle cells, which had been treated with VP or vehicle during stretch or no stretch for 6 hours. Initially, it was found that nuclei could not be efficiently isolated from fixed cells by any kind of homogenization at multiple settings. To maximize isolation of chromatin, it was found that isolation of nuclei was optimized by first washing cells in ice-cold PBS with protease inhibitors, then solubilizing unfixed cell membranes by incubating with $1 \%$ Triton X-100 in PBS for exactly 5 minutes on ice. Nuclei were pelleted by centrifugation at $4^{\circ} \mathrm{C}$ for 5 minutes at $3000 \times g$. The pelleted nuclei were gently washed at $4{ }^{\circ} \mathrm{C}$ and fixed in $1 \%$ paraformaldehyde in PBS for 10 minutes, after which fixation was immediately blocked with glycine/PBS. Nuclei were washed three times in PBS and placed at $-80^{\circ} \mathrm{C}$ until further processing. Nuclear pellets were sonicated on ice to attain optimal DNA fragmentation (400-bp fragment size). Debris was pelleted at $3000 \times g, 3$ minutes. Input DNA was isolated from the resulting chromatin in the supernatant by reversing cross-links, phenol extraction, and ethanol precipitation. The chromatin was immunoprecipitated as in the High-Sensitivity ChIP kit (Active Motif, Carlsbad, CA), using the YAP/WWTR1 or species control IgG antibody for immunoprecipitation. Cross-links were reversed with heat and $\mathrm{NaCl}$, then DNA isolated by phenol chloroform extraction. PCR primers were designed around the promoter of $B D N F$ (Table 1).

\section{Statistical Analysis}

Physiology data were analyzed using Welch's $t$-test, when variances were not equal, and $t$-test, with $P<0.05$ considered significant. All $t$-tests were performed as two-tailed, except where indicated in the text. Correlation analysis was performed by Pearson's correlation (using the stats package Hmisc on R) to identify parameters from physiological and expression data that correlate and cluster together. Expression data were analyzed initially using limma on $\mathrm{R}$, with an interquartile range of 0.9 , using the HTqPCR package. ${ }^{13}$ Correlation analysis of expression and physiology data were analyzed in both linear and logarithmic formats $[-$ delta $\mathrm{c}(\mathrm{t})]$ to facilitate comparisons. ${ }^{13}$ Correlations with $P<0.05$ and $R^{2}>0.40$ were selected as potential candidates and plotted graphically. In addition, $\mathrm{R}$, $R^{2}$, and $P$ values for correlations were plotted using $\mathrm{R}$ ggcorrplot packages to identify genes whose expression patterns were associated with physiological parameters. Post hoc Student's tests, Welch's $t$-tests, and analysis of variance of gene expression data were performed. For all tests, $P$ values were considered significant if $P<0.05$.

\section{Results}

Inhibiting DNA Methylation during Partial Bladder Outlet Obstruction Exacerbates Gross Bladder Hypertrophy, SMC Hypertrophy, and Loss of Smooth Muscle Differentiation Markers

PBO alone (without inhibitor) caused a significant increase in mean bladder mass $(P<0.005)$ and bladder/body mass ratio $(P<0.005)$ compared with sham animals (Figure 1, A and B). DAC-treated PBO animals showed further elevations in mean bladder mass $(P<0.05)$ and bladder/body mass ratio $(P<0.05$, one-tailed $t$-test $)$ versus $\mathrm{PBO}$ alone (Figure 1, A and B). Body mass at time of surgery varied from 250 to $300 \mathrm{~g}$, and body mass was not significantly altered (Supplemental Figure S4, A-F and $\mathrm{H}$ ). More important, DAC treatment had only minimal effects on final mass of sham bladders. Transnuclear cross-sectional SMC area was increased significantly during PBO, compared with sham $(P<0.01)$ (Figure $1, \mathrm{C}$ and $\mathrm{D})$. Consistent with bladder mass, the increase in obstruction-induced SMC area was significantly exacerbated by DAC treatment $(P<0.05)$ (Figure 1D). In contrast to increased SMC size, obstruction led to a twofold decrease in smooth muscle myosin marker fluorescence within SMC bundles $(P<0.05)$ (Figure $1, \mathrm{C}$ and $\mathrm{E})$. SM myosin signal was further dampened by $\mathrm{PBO}+$ DAC treatment versus sham (Figure 1, C and E).

\section{Inhibiting DNA Methylation during Partial Bladder Outlet Obstruction Exacerbates Obstruction-Induced Bladder Dysfunction}

PBO decreased maximal micturition fraction $(P<0.05)$ (Figure $1 \mathrm{~F}$ ), which is a surrogate for efficiency (completeness) of voiding. ${ }^{8}$ DAC treatment during PBO specifically decreased maximum and mean micturition fractions by greater than twofold versus PBO alone $(P<0.01)$ (Figure 1, $\mathrm{F}$ and $\mathrm{G}$, respectively). Residual volume and maximal bladder capacity increased significantly in both $\mathrm{PBO}$ and PBO-DAC groups compared with sham $(P<0.01)$ (Figure 1, H and I, respectively). Moreover, DAC treatment of $\mathrm{PBO}$ versus vehicle treatment of $\mathrm{PBO}$ significantly increased residual volume (6.88-fold, $P<0.05$ ) (Figure $1 \mathrm{H}$ ) and bladder capacity ( 2.75 -fold, $P<0.05$, by one-tailed $t$-test) (Figure 1I). DAC did not affect capacity or residual volume in sham-operated rats (Figure 1, H and I). Nonsignificant changes between $\mathrm{PBO}$ and PBO-DAC were observed in maximum voided volumes, total voided 


\section{A \\ C}
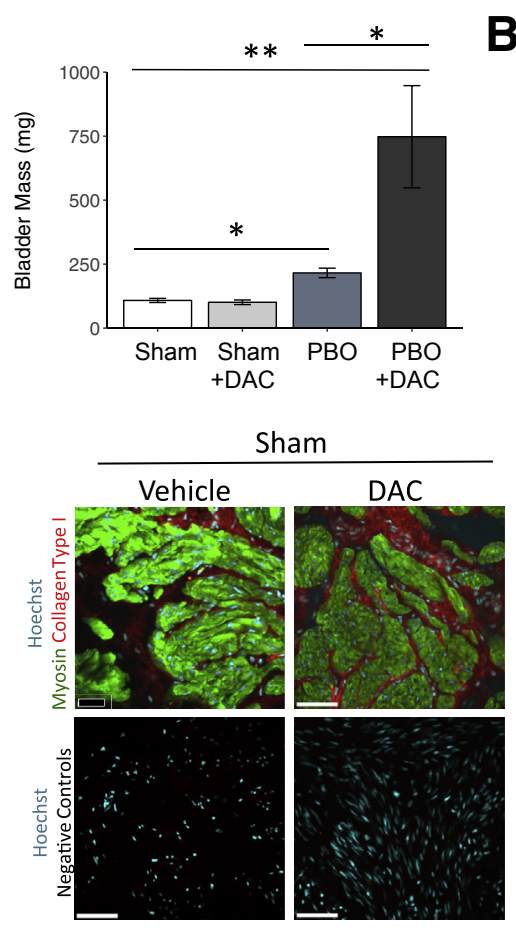

B
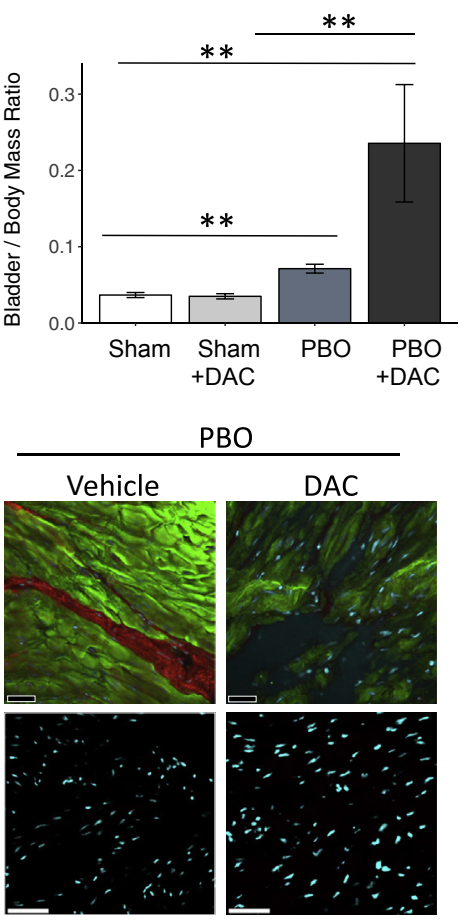

\section{D}

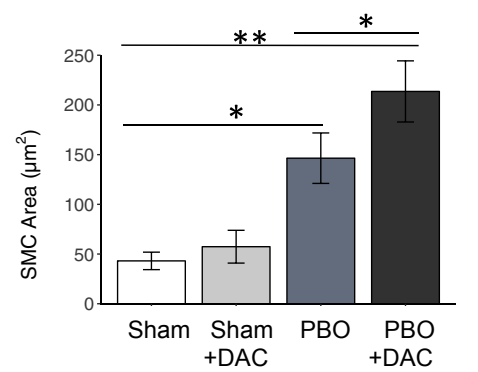

$\mathbf{F}$

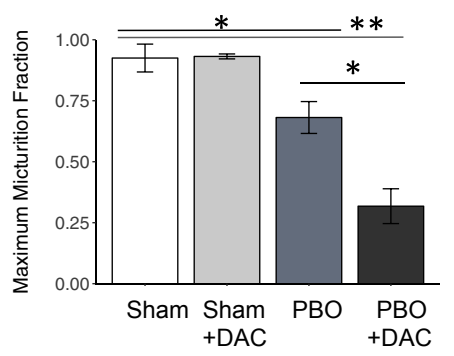

H

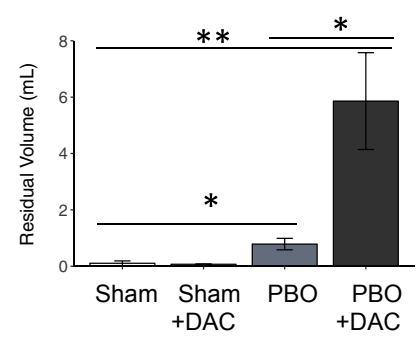

E

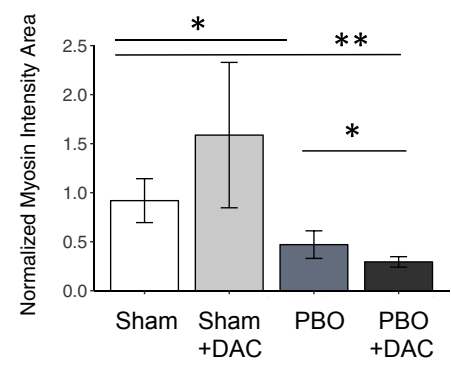

G
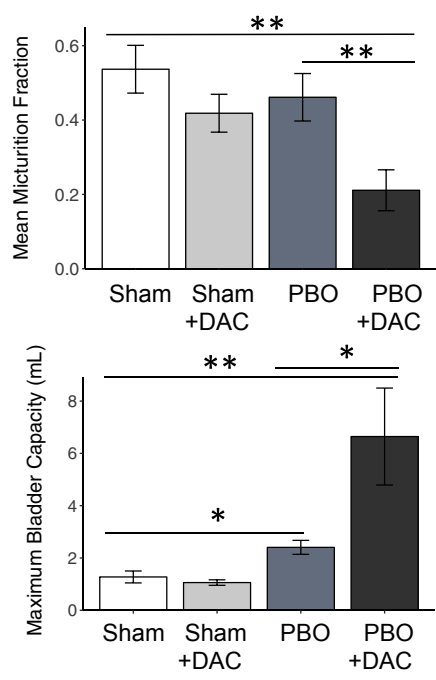

Figure 1 DNA methylation protects against smooth muscle hypertrophy and loss of function during partial bladder obstruction. A and B: Inhibition of DNA methylation by $1 \mathrm{mg} / \mathrm{mL}$ of 5-aza-2-deoxycytidine (or decitabine; DAC) leads to worsening of the physiological response to obstruction, including bladder mass $(\mathbf{A})$ and bladder/body mass ratios (B). C: Immunofluorescence staining of smooth muscle myosin heavy chain (green) was used to mark smooth muscle bundles in the detrusor. Collagen type I was also detected (red). Negative control images (primary antibodies absent, with secondaries included) shown for each represented sample. Representative images were taken. D: Cross-sectional smooth muscle cell (SMC) hypertrophy of cells containing circular nuclei (indicating the middle of the cell) has higher hypertrophy by cross section compared with sham. E: Myosin intensity normalized to areas of SMC show a decrease in myosin content by immunofluorescence detection. $\mathbf{F}$ and G: Contractile dysfunction during obstruction is restrained by DNA methylation. G-I: Changes in maximum (I) and mean (G) micturition fraction, and bladder capacity and residual urine volumes $(\mathbf{H})$ during partial bladder outlet obstruction are restrained by DNA methylation. $\mathbf{H}$ and $\mathbf{I}$ : Partial bladder outlet obstruction (PBO) increases residual volumes $(\mathbf{H})$ and maximum bladder capacity $(\mathbf{I})$, above sham or sham + DAC groups, whereas decitabine treatment of PBO animals (PBO + DAC) further elevates volumes in both residual volumes and maximum bladder capacity. Sample numbers are described in the animal model schematic (Supplemental Figure S2). $n=6$ samples for sham or sham+DAC (C); $n=10$ PBO or PB0+DAC (C). ${ }^{*} P<0.05,{ }^{*} P<0.01$. Scale bars: $52 \mu \mathrm{m}$ (white bars); $34 \mu \mathrm{m}$ (black bars). Original magnification, $\times 200$ (C). volumes, and wall thickness (Supplemental Figure S4). Other physiological parameters, such as frequency of voids or small voids, and mean voided volumes (Supplemental Figure S4) showed only trends toward reversal of PBO effects by DAC treatment. The mean voided volume of PBO alone showed a trend toward increasing 1.5-fold higher than PBO-DAC $(P<0.10)$ (Supplemental Figure S4G). By Pearson's correlations, neither bladder kinetics nor hyperactivity (ratio of number of small voids/number of total voids and number of small voids) correlated with gross or 
Table 2 Significant Changes in Gene Expression Comparing Sham, Obstruction (PBO), and PBO + DAC Treatment by High-Throughput qPCR

\begin{tabular}{|c|c|c|c|}
\hline Gene & $P$ value & $\Delta \Delta C t$ & $\mathrm{FC}$ \\
\hline \multicolumn{4}{|c|}{ Sham versus PBO } \\
\hline Atp2a2 & $2.50 \times 10^{-2}$ & 2.554 & 0.17 \\
\hline$B d n f$ & $1.13 \times 10^{-2}$ & -3.396 & 10.53 \\
\hline Cond1 & $3.67 \times 10^{-2}$ & 2.579 & 0.17 \\
\hline$D b p$ & $1.21 \times 10^{-2}$ & 2.569 & 0.17 \\
\hline Dnmt1 & $2.82 \times 10^{-2}$ & 3.119 & 0.12 \\
\hline Dnmt3b & $1.26 \times 10^{-2}$ & 4.4315 & 0.05 \\
\hline Galr2 & $3.52 \times 10^{-2}$ & 2.6415 & 0.16 \\
\hline Gem & $2.18 \times 10^{-2}$ & 2.549 & 0.17 \\
\hline Kcnb2 & $1.98 \times 10^{-2}$ & 2.839 & 0.14 \\
\hline Kcnk1 & $2.99 \times 10^{-2}$ & 2.684 & 0.16 \\
\hline Kenma1 & $2.24 \times 10^{-2}$ & 3.4765 & 0.09 \\
\hline Chrna10 & $3.36 \times 10^{-2}$ & 2.339 & 0.20 \\
\hline RIMS1 & $3.24 \times 10^{-2}$ & 2.2515 & 0.21 \\
\hline Thrb & $4.23 \times 10^{-3}$ & 3.574 & 0.08 \\
\hline$T s c 22 d 3$ & $1.95 \times 10^{-2}$ & 3.5665 & 0.08 \\
\hline Ctgf & $4.55 \times 10^{-2}$ & -2.206 & 4.61 \\
\hline Chrna5 & $4.26 \times 10^{-2}$ & 3.944 & 0.06 \\
\hline Мeсp2 & $4.35 \times 10^{-2}$ & 2.444 & 0.18 \\
\hline \multicolumn{4}{|c|}{ Sham versus $\mathrm{PBO}+\mathrm{DAC}$} \\
\hline$B d n f$ & $5.04 \times 10^{-5}$ & -6.33 & 80.45 \\
\hline Ctgf & $2.20 \times 10^{-3}$ & -3.66 & 12.64 \\
\hline Dnmt3b & $2.98 \times 10^{-2}$ & 3.7775 & 0.07 \\
\hline Kcnb2 & $3.50 \times 10^{-2}$ & 2.5325 & 0.17 \\
\hline Thrb & $4.34 \times 10^{-2}$ & 2.3775 & 0.19 \\
\hline \multicolumn{4}{|c|}{$\mathrm{PBO}$ versus $\mathrm{PBO}+\mathrm{DAC}$} \\
\hline Adra1d & $2.11 \times 10^{-2}$ & -3.0165 & 8.09 \\
\hline$B d n f$ & $2.55 \times 10^{-2}$ & -2.934 & 7.64 \\
\hline
\end{tabular}

The only gene significantly altered in mRNA expression among all three comparisons was $B d n f$. Ctgf was significantly different in two comparisons (sham-PBO and sham-PBO + DAC). $P<0.05$ was considered significant $(t-$ tests by limma).

CTGF, connective tissue growth factor; DAC, 5-aza-2'-deoxycytidine (or decitabine); FC, fold change; PB0, partial bladder outlet obstruction; $\mathrm{PPCR}$, real-time quantitative PCR.

SMC hypertrophy (Supplemental Figure S5 and Supplemental Table S2). Rather, correlations were observed between hyperactivity, bladder capacity, and residual volumes. More important, the maximum micturition fraction correlated inversely with residual volume, bladder capacity, and gross hypertrophy.

\section{BDNF, CYR61, and CTGF Expression Levels Correlate with Bladder Pathophysiology}

mRNA expression of multiple candidate genes was examined by custom HT-qPCR array in sham, PBO, and PBO + DAC groups. PBO caused a variety of changes in candidate gene expression, with Bdnf appearing as the most significantly increased mRNA among all comparisons $(P<0.05)$ (Table 2). Principal component analysis revealed that each group formed separate clusters (Figure 2A). The greatest divergence between treatment groups was seen in principle component 2 (Table 3), differentiating the $\mathrm{PBO}+\mathrm{DAC}$ groups from the $\mathrm{PBO}$ and sham groups. Two genes contributed greatly to the weight of principle component 2 : Bdnf and $\operatorname{Ctgf}$ (Table 3).

Pearson's correlation analysis of the entire data set of HTqPCR genes alongside the physiological parameters revealed that expression of three genes, Bdnf, Ccnl, and Ctgf, cosegregated (Supplemental Figure S6). Ctgf highly correlated with $\operatorname{Bdnf}\left(P<10^{-6}\right)$, and strongly correlated with maximum micturition fraction and SMC area (Pearson's correlations, $R^{2}>0.4, P<0.05$ ) (Figure $2 \mathrm{~B}$ ). Bdnf expression was coordinately linked to alterations in SMC hypertrophy, maximum bladder capacity, mean and maximum micturition fraction, and residual volume (Pearson's correlations, $R^{2}>0.4$, $P<0.01$ ) (Figure 2B). Moreover, correlations between $B d n f$ and gross muscle hypertrophy, bladder mass or bladder/body mass ratio, were significant $\left(R^{2}>0.25, P<0.01\right)$ (Supplemental Figure S7, A and B). In addition, bladder dysfunction showed a trend in correlating with two extracellular matrix genes, Lox and $F n l\left(R^{2}>0.4, P \leq 0.20\right)$ (Supplemental Figure S6). Fnl, a known YAP/WWTR1 target, was up-regulated in both $\mathrm{PBO}$ and $\mathrm{PBO}+\mathrm{DAC}$ $(P<0.05)$ (Supplemental Figure S6), whereas Lox showed a trend in up-regulation only with $\mathrm{PBO}+\mathrm{DAC}$ treatment. $F n 1$ also contributed to the principle component 2 , with the third highest eigenvalue (Table 3). In contrast to Bdnf, Cyr61+ Ctgf, Lox, and Fnl, most of the candidate genes tended to be down-regulated in the $\mathrm{PBO}+\mathrm{DAC}$ group versus $\mathrm{PBO}$ vehicle (Supplemental Figure S8). The Bdnf regulator methyl CpG binding protein 2 (MECP2) and alpha-1A adrenergic receptor (Adra1d) were up-regulated during obstruction but did not show any significant change with DAC treatment (Supplemental Figure S9). Although Bdnf, Cyr61, and Ctgf expression are well known to increase during obstructive myopathy in the bladder, this report shows their coregulation and cosegregation with physiological bladder dysfunction.

To confirm the dysregulation of the two most significantly altered genes, Bdnf and Ctgf, conventional real-time quantitative PCR was performed on cDNA from a larger set of samples. Bdnf and Ctgf were up-regulated 11.3- and 2.4fold, in PBO alone versus sham, respectively $(P<0.05)$ (Figure 2, C and D). Ctgf and Bdnf expression levels were further amplified during $\mathrm{PBO}+\mathrm{DAC}$ versus $\mathrm{PBO}$ in the confirmation data set $(P<0.05)$ (Figure 2, C and D). Ctgf mRNA was increased 27 -fold in PBO + DAC versus sham $(P<0.05)$. Bdnf mRNA showed the highest obstructioninduced up-regulation with decitabine treatment, 100-fold above sham $\left(P<0.05\right.$, using $2^{-\Delta \Delta \mathrm{Ct}}$ linear values; and $P<0.0001$, using $\Delta \mathrm{Ct}$ values). In sham plus DAC, these genes did not significantly change compared with sham plus vehicle. Using the larger confirmation data set, Bdnf expression correlated inversely with voiding efficiency (residual volume, micturition fraction, bladder capacity, $R^{2}>0.4, P<0.005$ ) (Supplemental Figure S7, C-E). By enzyme-linked immunosorbent assay, BDNF protein levels from $\mathrm{PBO}$ or $\mathrm{PBO}+\mathrm{DAC}$ increased above sham 
A

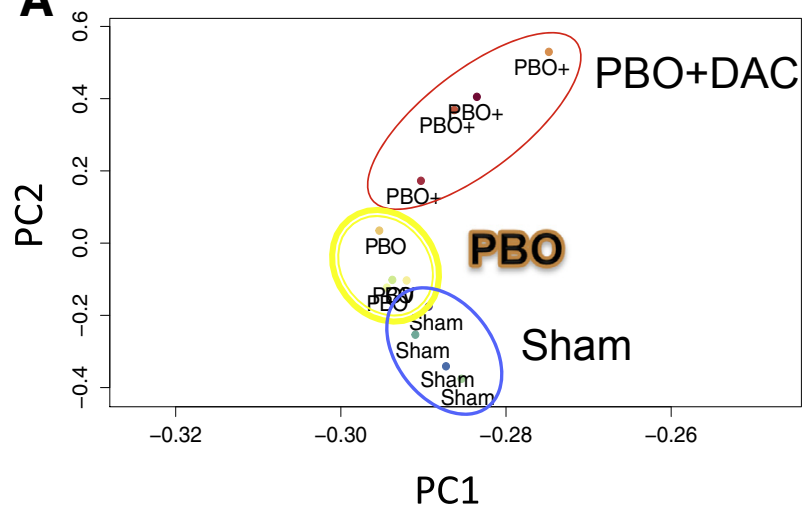

B
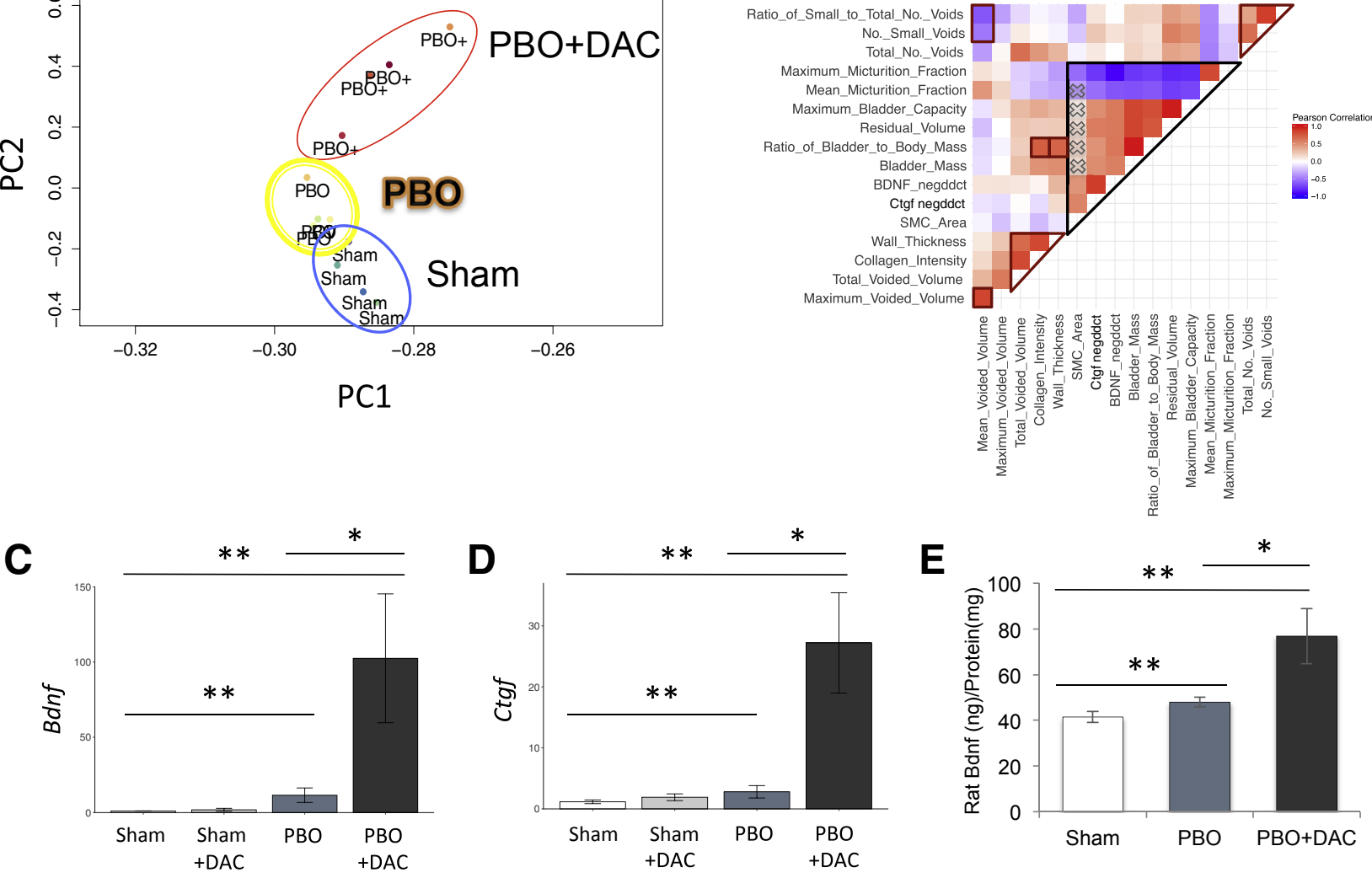

D

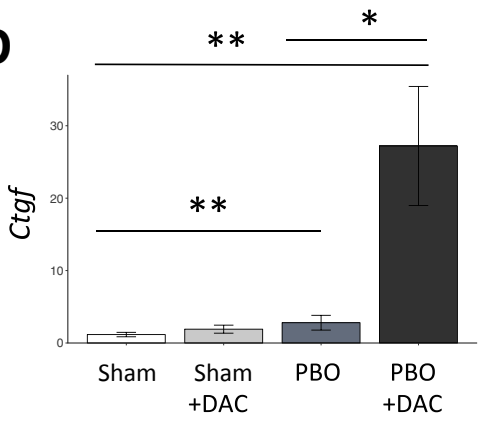

E

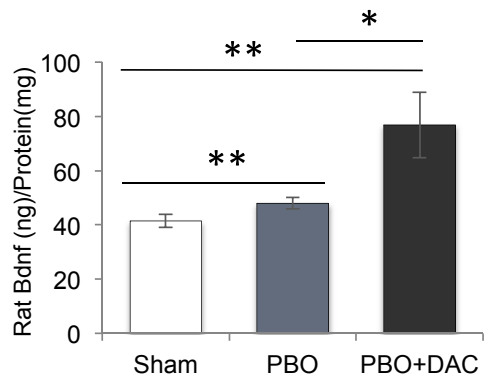

$\mathbf{F}$

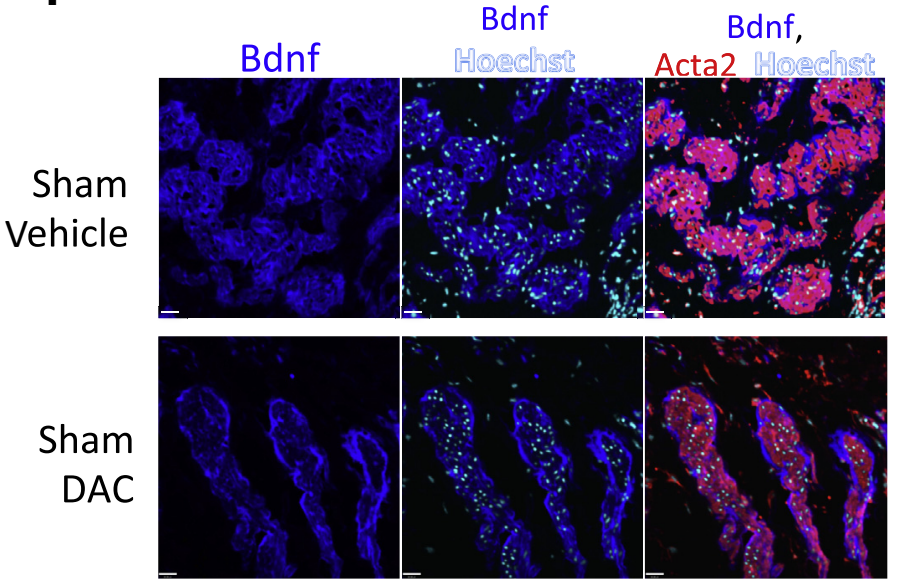

PBO
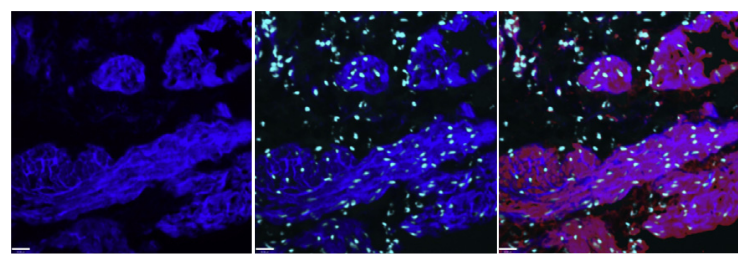

PBO

DAC

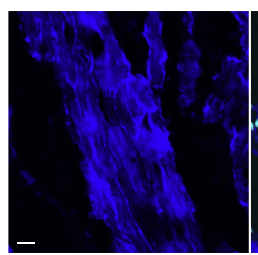

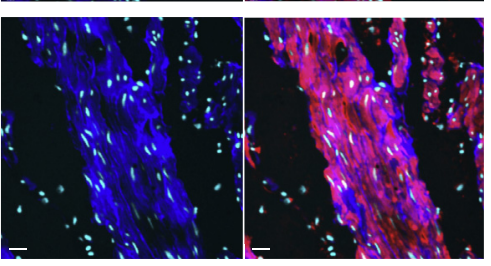

Figure 2 Analysis of high-throughput real-time quantitative PCR array data uncovers a group of genes (Bdnf, Cyr61, Ctgf) that cluster together and correlate with pathophysiologic changes. A: Principal component analysis of gene expression clusters the surgical and treatment groups with minimal overlap. The greatest divergence appears in principal component 2 (PC2). The coordinates of PC1 + PC2 are described in Table 2 and show that two of the most significantly altered genes are Bdnf and Ctgf. B: Pearson correlations show a high degree of correlation between the negative $\Delta \Delta C$ t values for Bdnf and Ctgf with micturition fraction, smooth muscle cell (SMC) area, and each other, brain-derived neurotrophic factor (BDNF) correlates with bladder mass, bladder/body weight ratio, and maximum bladder capacity $(P<0.05)$. Black triangle indicates significant correlations $(P<0.05)$, excluding those correlations with $X$, which are nonsignificant. Significant correlations between physiological parameters are depicted in Supplemental Figure S5. Red triangles and squares indicate other significant correlations between pathophysiologic parameters. $\mathbf{C}$ and $\mathbf{D}$ : $B \operatorname{dnf}(\mathbf{C})$ and $\mathbf{C t g f}(\mathbf{D})$ mRNA expression differs significantly between the partial bladder outlet obstruction (PBO) and PBO + 5-aza-2-deoxycytidine (or decitabine; DAC) as well as sham versus PBO or PBO + DAC. E: Enzyme-linked immunosorbent assay results from protein lysate of whole bladder reveal an increase in BDNF protein in $\mathrm{PBO}$ or $\mathrm{PBO}+\mathrm{DAC}$. As bladders were washed before protein extraction and bound, intracellular BDNF was preferentially detected. F: BDNF immunofluorescence intensity is increased during obstruction and exacerbated by DAC. Representative confocal immunofluorescence images show that BDNF is present in the urothelium and detrusor, although only detrusor BDNF appears to increase during $\mathrm{PBO}$ or $\mathrm{PBO}+$ DAC. Decitabine treatment ( $1 \mathrm{mg} / \mathrm{kg}$ per day) for 6 weeks during PBO increases BDNF intensity around smooth muscle bundle cells of the detrusor, which are indicated by myosin staining. Hoechst (light bluewhite) staining was used to define the nucleus of the cells. $n=4$ (A, $\mathrm{PB} 0+\mathrm{DAC}$ and sham) $; n=3$ (A, PB0 alone). ${ }^{*} P<0.05,{ }^{*} P P<0.01$. Scale bars $=24 \mu \mathrm{m}(\mathbf{F})$. Acta2, smooth muscle actin. 
Table 3 Coordinates of PCs 1 and 2 of the 20 Most Significantly Changed Genes in the 3 Groups [Obstruction, Obstruction Plus 5-Aza-2-Deoxycytidine (or Decitabine), and Sham]

\begin{tabular}{llr}
\hline Gene & PC1 & PC2 \\
\hline Ctgf & 0.023 & 0.305 \\
Bdnf & 0.024 & 0.304 \\
Adra1d & 0.139 & 0.076 \\
RIMS1 & 0.131 & -0.129 \\
Fn1 & 0.121 & 0.180 \\
Gys1 & 0.152 & 0.008 \\
Socs3 & 0.135 & 0.123 \\
Des & 0.148 & 0.033 \\
Ryr2 & 0.132 & 0.055 \\
Galr2 & 0.149 & -0.026 \\
Ccnd1 & 0.156 & -0.020 \\
Chrna5 & 0.126 & -0.067 \\
Traf6 & 0.150 & -0.037 \\
Gem & 0.150 & -0.083 \\
Dbp & 0.129 & -0.177 \\
Mmp7 & 0.013 & 0.163 \\
Cyr61 & 0.086 & 0.173 \\
Mmp9 & 0.002 & 0.241 \\
Srf & 0.153 & -0.046 \\
Atp2a2 & 0.148 & -0.099 \\
\hline
\end{tabular}

Genes with $\mathrm{PC}$ analysis values $>0.160$ are in bold, including $B d n f$, Ctgf, and Cyr61. Data is displayed in Figure 2.

PC, principal component.

$(P<0.05)$ (Figure 2E). Likewise, BDNF from PBO + DAC was also $60 \%$ higher than PBO $(P<0.01)$. Similarly, immunofluorescence revealed that BDNF was up-regulated in PBO and PBO-DAC groups, particularly around smooth muscle cells and bundles identified by smooth muscle actin (Figure 2F).

\section{DNA Methylation Regulates Bdnf and Ctgf Expression in Cultured BSMCs}

Because DNA methylation has been reported to correlate with Bdnf expression during rat partial obstruction, the response of BDNF or CTGF transcription to inhibition of DNA methylation in human BSMCs was determined. In vitro treatment of resting BSMCs with decitabine for 24 hours increased baseline BDNF and CTGF expression $(P<0.01)$ (Figure 3, A and B). DNA methylation of the $B D N F$ locus was also explored using pyrosequencing of genomic DNA from in vivo obstruction samples. Only three sites of 17 analyzed demonstrated significant alterations in methylation by one-tailed $t$-test $(P<0.05)$, between sham, $\mathrm{PBO}$, and PBO + DAC groups (data not shown). However, the DAC-induced changes were small, $0.3 \%$ to $0.9 \%$ changes in cytosine-guanosine dinucleotide $(\mathrm{CpG})$ methylation, suggesting that regulation of these genes by DNA methylation may be occurring downstream of other epigenetic activity.
Stretch-Induced Gene Expression of BDNF, CYR61, and CTGF mRNA Requires YAP/WWTR1 Activity

Because a $0.9 \%$ change in DNA methylation of the Bdnf promoter is unlikely to lead to the observed 8.9-fold decitabineinduced up-regulation of Bdnf in PBO rats, the pathways that may be involved in up-regulation of the three clustered genes, $B D N F, C Y R 61$, and $C T G F$, were analyzed in silico. The YAP/ WWTR1 pathway was identified along with the transcription factor binding site transcriptional enhancer activator domain transcription factor 1 (TEAD1) in TOPGO and OPOSSUM 3.0 (Supplemental Tables S3 and S4). Other pathways were identified for these three genes, including macrophage integrin signaling, extracellular matrix protein and proteoglycans, hypertrophy, mitogen-activated protein kinase signaling, RhoA signaling, transforming growth factor- $\beta$, neurotrophic signaling, and Wnt signaling. Within the highly conserved human and mouse promoters of BDNF, CYR61, and CTGF, OPOSSUM 3.0 identified many motifs that were common to these three genes compared with the background set of genes. Particularly, TEAD1 consensus binding regions were identifiable in $C Y R 61+C T G F, L O X$, and the large $B D N F$ gene locus (Supplemental Table S4). The TEAD1 transcription factor binding site is previously unexplored in BSMCs and bladder obstruction. Other transcription factor binding site identified included sites previously explored by other researchers, including NF- $\kappa \mathrm{B}$, CCAAT-enhancer-binding proteins, and nuclear factor of activated $\mathrm{T}$ cells. TEADs bind DNA with cofactors YAP/WWTR1, which comprise a crucial part of the Hippo growth regulatory pathways.

Because YAP/WWTR1 localization to the nucleus is needed for function, nuclear YAP/WWTR1 expression and localization were evaluated during mechanical bladder strain injury, an in vitro functional surrogate for bladder obstruction. ${ }^{10,11}$ Using an antibody that reacts to both YAP and WWTR1, YAP and WWTR1 localization to the nucleus was significantly increased in mechanically strained human BSMCs at 6 hours (3.3-fold, $P<0.05$ ) (Figure 3, C and D). Time points earlier than 6 hours failed to show a significant increase in localization.

We then tested if transcription responses to 8 hours of mechanical strain affected BDNF and CTGF mRNA in human BSMCs. This time point was selected to allow for transcription of target genes after nuclear localization of YAP/ WWTR1 at 6 hours. Mechanical strain significantly induced mRNA of BDNF (1.5-fold, $P<0.05$ ) (Figure 3E) and CTGF (2.4-fold, $P<0.005$ ) (Figure 3F). To examine YAP/WWTR1 dependency of BDNF and CTGF expression, the YAP/ WWTR1 inhibitor VP was used to treat BSMCs during mechanical strain. VP reduced strain-induced and basal CTGF mRNA expression $(P<0.001)$ (Figure $3 \mathrm{~F}$ ) and straininduced mRNA expression of BDNF (1.9-fold, $P<0.01$ ) (Figure 3E), supporting a role for YAP/WWTR1 signaling in BDNF and CTGF expression in vitro. In contrast to this, the SMC differentiation marker, MYH11, was increased by VP $(P<0.01)$ (Figure 3G) during both stretch and no stretch. 

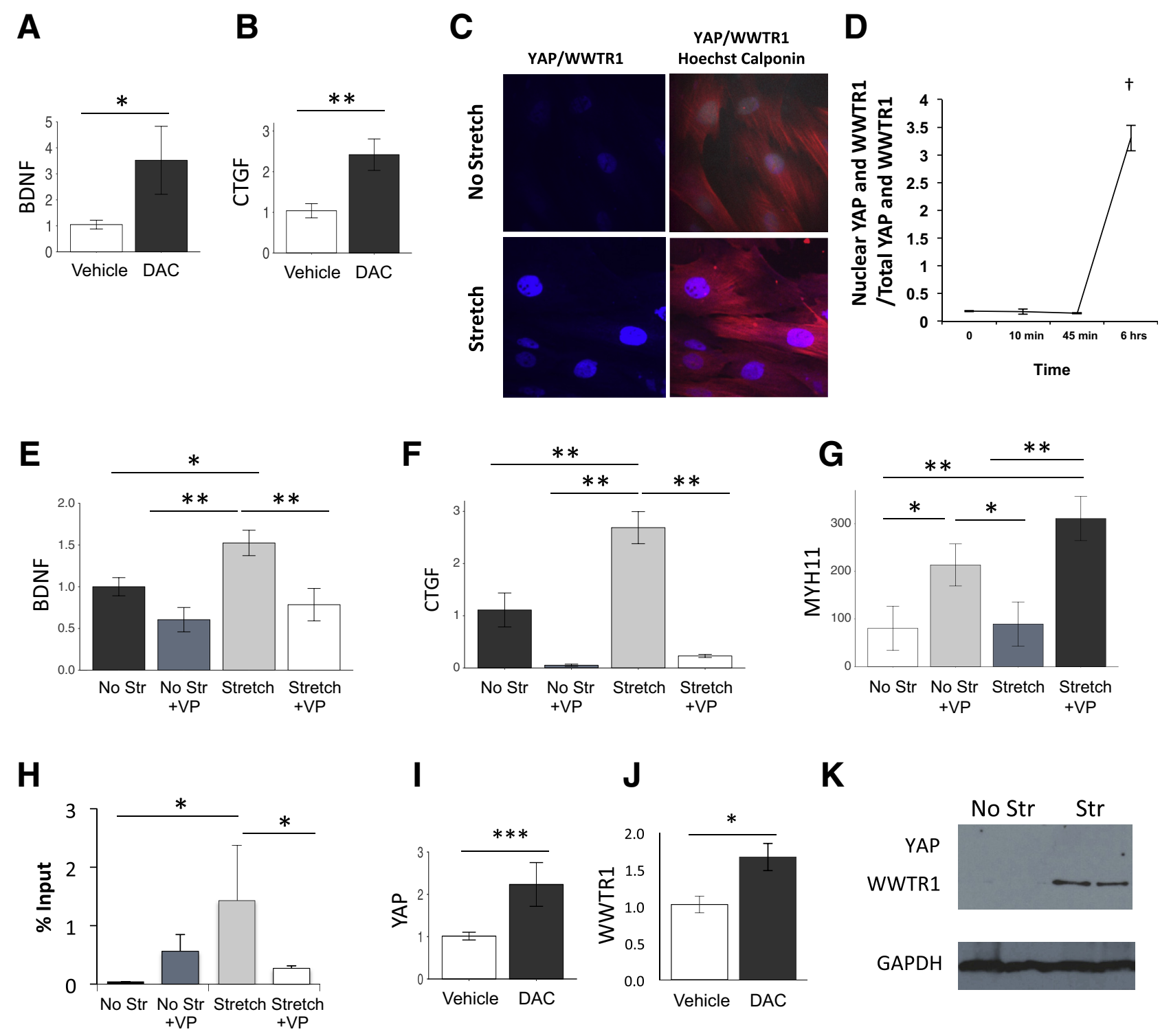

Figure 3 Brain-derived neurotrophic factor (BDNF) and CTGF mRNAs are repressed by DNA methylation but increased by mechanical strain. A and B: Expression (mRNA) of BDNF (A) and CTGF (B) is significantly constrained by DNA methylation, as loss of DNA methylation by $2 \mu$ mol/L 5-aza-2-deoxycytidine (or decitabine; DAC) treatment increases BDNF and CTGF mRNA expression, 3.5- and 2.4-fold, respectively. Human bladder smooth muscle cells (BSMCs) seeded onto type I collagen BioFlex plates were treated for 1 hour with vehicle or verteporfin (VP). C-K: Cells were then stretched on the Flexcell 3000 system for 8 hours $(\mathbf{C}-\mathbf{G}), 6$ hours $(\mathbf{H}$ and $\mathbf{K}$ ), or up to 24 hours (I and $\mathbf{J})$, using a ramping program of increasing mechanical strain to $5 \%$ elongation. $\mathbf{C}$ and $\mathbf{D}$ : Immunocytochemistry for YAP/WWTR1 shows increased nuclear localization after mechanical strain in bladder smooth muscle. E-G: Cells were analyzed by real-time quantitative RT-PCR for Bdnf, Ctgf, and Myh11. Discrete mechanical stimulation of BSMCs in vitro significantly increases mRNA of Bdnf 1.5-fold and of CTGF 2.4-fold. In stretched cells, Bdnf and CTGF are reduced by VP 1.9- and 11.6-fold, respectively. G: MYH11 shows the opposite pattern. H: Chromatin Immunoprecipitation/PCR was performed on human BSMCs after 7 hours of mechanical strain, compared with no stretch and stretch + VP. YAP binding to the BDNF promoter increases with mechanical strain and is blocked by verteporfin, an inhibitor that sequesters YAP in the cytoplasm. I and J: Levels of Yap and WWTR1 mRNA in human bladder smooth muscle cells are also dependent on DNA methylation as WWTR1 increases 1.8-fold with decitabine treatment. K: Expression of WWTR1 increases during bladder smooth muscle stretch, as examined by Western blotting. $n=4$ (H). ${ }^{*} P<0.05,{ }^{* *} P<0.01$, and ${ }^{* * *} P<0.001$; ${ }^{\dagger} P<0.05$ versus all other time points. GAPDH, glyceraldehyde-3-phosphate dehydrogenase. Min, minutes; Str, stretch.

\section{Mechanical Strain Increases YAP/WWTR1 Binding to the Bdnf Locus in BSMCs}

To test for a YAP/WWTR1 association at the $B D N F$ promoter, chromatin immunoprecipitation/PCR was performed. Binding of YAP/WWTR1 to the region before the second promoter increased during mechanical strain (33-fold increase, $P<0.05$, by one-tailed $t$-test) (Figure $3 \mathrm{H}$ ). This binding was dependent on YAP/ WWTR1, because their inhibitor, VP, greatly reduced stretch-induced binding at this locus (5.3-fold reduction, $P<0.05$, one tailed). 


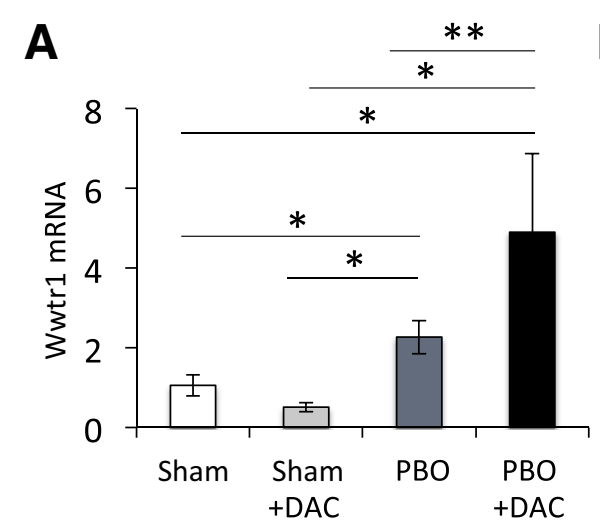

B

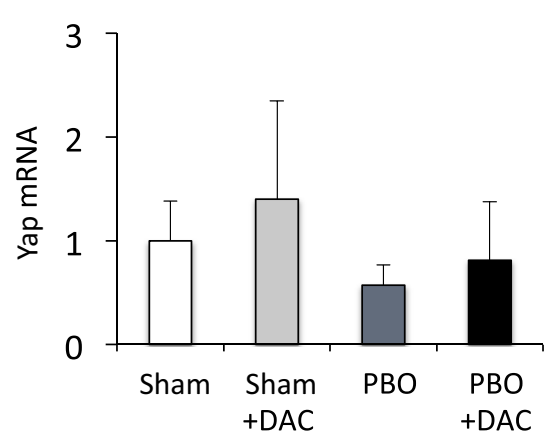

C

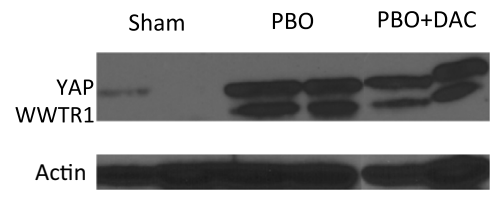

D

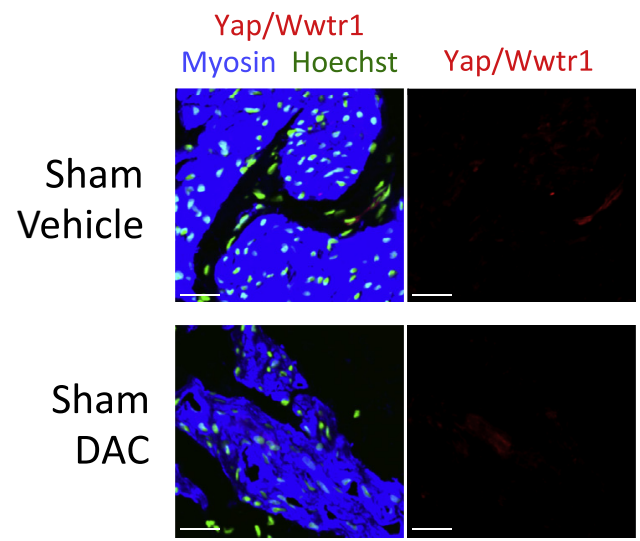

PBO
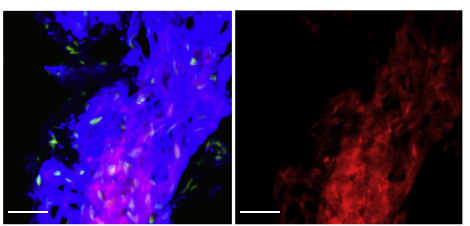

PBO

DAC
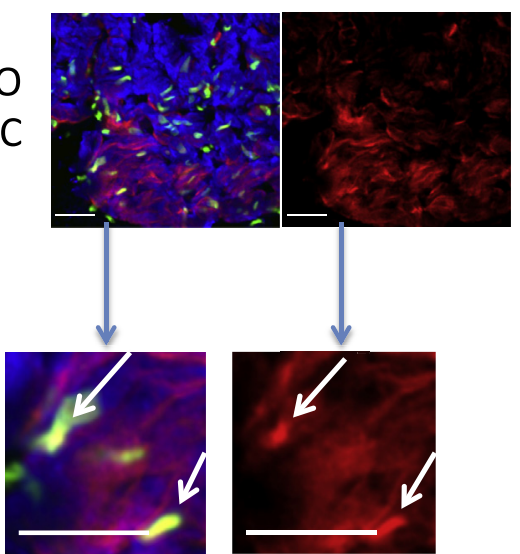
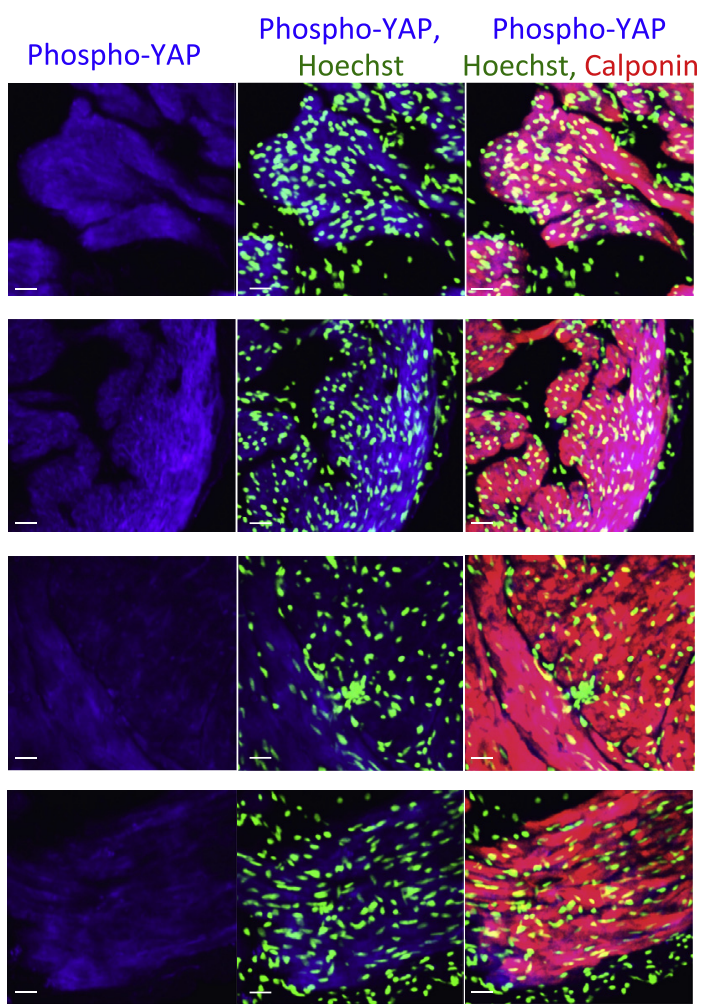

Figure 4 In vivo expression of YAP and WWTR1 is repressed by DNA methylation. A and B: In vivo, transcription of WWTR1 during partial bladder outlet obstruction (PB0) 5-aza-2-deoxycytidine (or decitabine; DAC) treatment significantly increases (A), whereas YAP only shows nonsignificant trends in mRNA fluctuations (B). C: Western blotting for YAP and WWTR1 demonstrates an increase in total bladder levels of YAP and WWTR1. D: Immunofluorescence staining of representative cryosections (7 $\mu \mathrm{m}$ thick) with a YAP/WWTR1-specific antibody reveals that YAP/WWTR1 appear to be increased during obstruction and localized to the nucleus more during decitabine treatment in bladder smooth muscle (identified by smooth muscle myosin). Left panels: Intensity of nuclear compared to cytoplasmic YAP/WWTR1 immunofluorescent signal was quantified on Volocity software version 6.2. Right panels: Phosphorylated YAP signal decreases in the smooth muscle compartment. D: Insets: White arrows: nuclear-localized YAP/WWTR1 signal. ${ }^{*} P<0.05,{ }^{*} P<0.01$. Scale bar $=24$ $\mu \mathrm{m}$ (D, main images and insets). 
Inhibiting DNA Methylation Up-Regulated WWTR1 and YAP Expression and Nuclear Localization but Not TEAD Expression

Given the effect of DNA methylation inhibition on transcription of $C T G F$ and Bdnf in rat obstruction in vivo and the role of the YAP/WWTR1 inhibition on their expression in vitro, it was speculated that DNA methylation would regulate $C T G F$ and $B d n f$ indirectly through $Y A P$ and WWTRI expression during in vitro culture. In vitro treatment of BSMCs with DAC led to an increase in both YAP (2.2-fold, $P<0.05$ ) (Figure 3I) and WWTR1 (1.67-fold, $P<0.01$ ) (Figure 3J) expression. Finally, 6 hours of mechanical stretch increased WWTR1 protein expression in human BSMCs by Western analysis as well (Figure 3K).

In vivo, $\mathrm{PBO}$ increased Wwtr1 2.3-fold above sham levels $(P<0.05)$ (Figure 4A), whereas $\mathrm{PBO}+$ DAC showed a trend toward increasing Wwtrl levels above $\mathrm{PBO}$ $(P<0.10)$. Nevertheless, PBO + DAC increased 4.9 -fold compared with sham levels, with a $t$-test of $P<0.05$ using $\Delta \mathrm{Ct}$ values. Yap expression was unchanged in mRNA expression (Figure 4B). TEADs are transcription factors that associate with YAP/WWTR1. In vivo, only Tead-1 and Tead-4 were detectable, but both remained unchanged during either obstruction or treatment (Supplemental Figure S10).
A

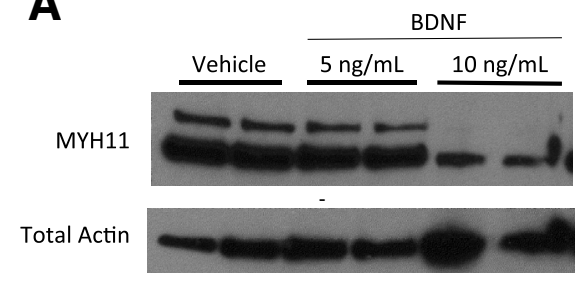

D

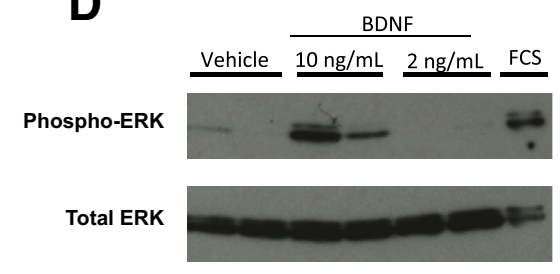

$\mathbf{F}$

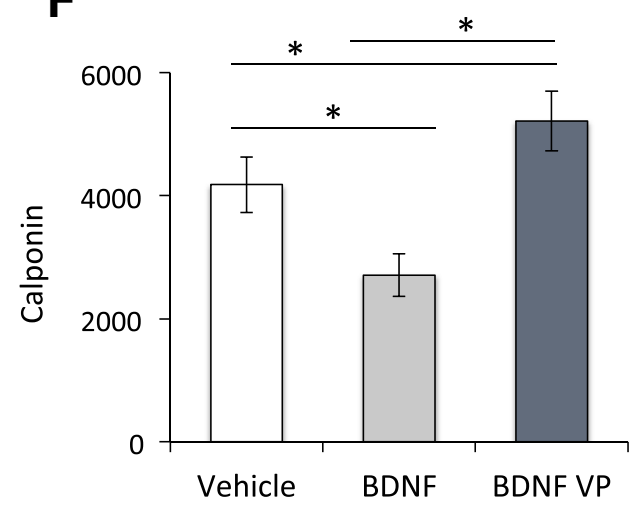

B

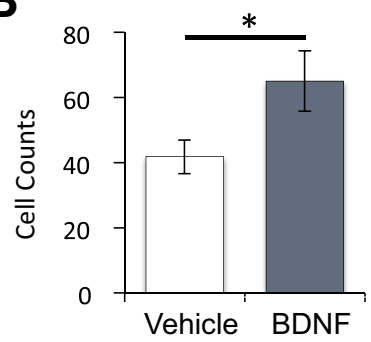

$\mathbf{E}$

Phospho-S6K

Total S6K

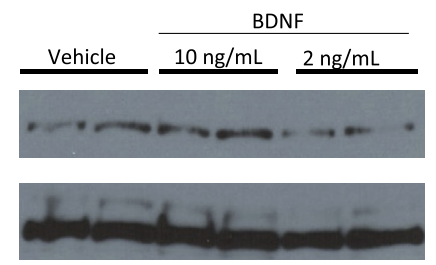

G

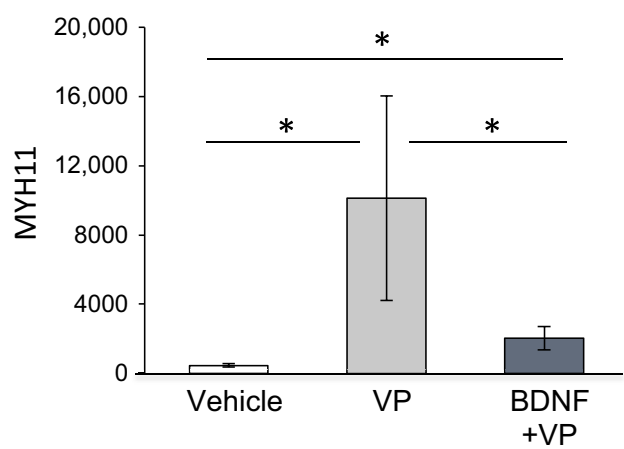

Figure 5 Brain-derived neurotrophic factor BDNF) directly reduces smooth muscle cell (SMC) differentiation and growth. A: Treatment of human bladder SMCs with exogenous recombinant BDNF ( $10 \mathrm{ng} / \mathrm{mL}$; Alamone Labs) leads to a decrease in smooth muscle myosin after 48 hours. B: After 24 hours, BDNF significantly increases the number of bladder SMCs by cell counting of more than three fields for four wells per treatment. C: After 48 hours, BDNF significantly increases proliferative marker PH3 in nuclei of bladder SMCs. BDNF directly affects SMC growth signaling. D and E: Exogenous BDNF increases extracellular signal-regulated kinase (ERK) and S6 kinase (S6K)1 signaling by Western blotting for phosphorylated ERK1/2 and S6K1, respectively. Total ERK1/2 and total S6K1, respectively, were used as reference proteins. ${ }^{15} \mathbf{F}$ and $\mathbf{G}$ : BDNF and verteporfin interact to affect smooth muscle marker expression in human bladder smooth muscle after 24 hours. Human bladder SMCs plated on denatured collagen were treated for 24 hours with $10 \mathrm{ng} / \mathrm{mL}$ BDNF, verteporfin (VP; $0.1 \mu \mathrm{mol} / \mathrm{L}$ ), or VP + BDNF. F and G: Cells were fixed in 4\% paraformaldehyde and immunofluorescently stained for calponin (F) or smooth muscle myosin (G). Intensity of confocal images was analyzed on Volocity software. G: BDNF decreases calponin expression. In contrast, VP augments myosin expression in bladder SMCs, which is reduced by BDNF treatment. ${ }^{*} P<0.05$. FCS, fetal calf serum. 
Because protein degradation and nuclear localization play crucial roles in Yap and Wwtr1 function, Western blots and immunofluorescence were used to examine Yap and Wwtr1 quantity and localization in detrusor muscle (myosin positive) of sham or obstructed bladders with vehicle or decitabine (Figure 4, C and D). By Western blot, Yap and Wwtr1 expression could be differentiated in vivo, which demonstrated a moderate level of basal Yap and elevated Yap and Wwtrl with obstruction with or without DAC (Figure 4C). Phosphorylated forms did not show high signal on the blots. Minimal immunofluorescence staining in sham or sham + DAC treated bladders was observed (Figure 4D). In PBO with vehicle, both cytoplasmic and nuclear expression was increased above sham levels. In contrast, nuclear localization was more prominent in DAC-treated PBO bladders, with higher-intensity signal in nuclei located in SMC bundles (Figure 4D). Minimal to no staining of cells between muscle bundles was noted in all groups. Conversely, the detection of the degraded form of YAP by immunofluorescence was minimal in obstruction groups with either vehicle or decitabine.

\section{BDNF Protein Alters BSMC Growth, Growth Signaling Pathways, and SMC Marker Expression}

Because Myh11 immunofluorescence was down-regulated, whereas BDNF increased during $\mathrm{PBO}$ and $\mathrm{PBO}$ plus DAC (Figure 1), it was determined if the SMC phenotype (SMC growth and SMC marker expression) was affected by treatment with exogenous recombinant BDNF (Figure 5). Human BSMCs were plated on tissue culture plastic (Figure 5, A-E) or denatured type I bovine collagen (Figure 5, F and G) at $4 \times 10^{4}$ cells $/ \mathrm{mL}$, left to attach overnight, and serum starved for 24 hours. By Western analysis, the highest tested dosage of BDNF $(10 \mathrm{ng} / \mathrm{mL})$ led to a reduction of smooth muscle myosin heavy chain (Figure 5A). In addition, BDNF increased growth of BSMCs, with cell counts 1.55 -fold increased $(P<0.05)$
(Figure 5B). Expression of the proliferative marker phospho-histone-3 also increased $13 \%$ in response to BDNF $(P<0.05)$ (Figure 5C). Moreover, S6 kinase [mammalian target of rapamycin (mTOR)] and extracellular signalregulated kinase pathways, which have been previously implicated in mechanistic regulation of BSMC growth, ${ }^{11,12}$ were activated by high doses of BDNF (Figure 5, D and E).

\section{Verteporfin-BDNF Antagonism Leads to Altered SMC Phenotype}

Although VP treatment increased Myh11 expression (Figure 5G), VP also reduced BDNF expression (Figure 3E). To examine possible interactivity of BDNF with YAP/WWTR1 induction of SM markers, VP was added to exogenous BDNF-treated cells (Figure 5, F and G). Damaged matrix, which is induced during overdistension and stretch of bladder smooth muscle, ${ }^{11,12}$ is another surrogate of in vivo obstruction. BDNF treatment reduced protein expression of smooth muscle marker, calponin, on damaged matrix $(P<0.05)$ (Figure 5F). Also, Myh11 expression was affected in a different way: VP increased Myh11 expression in cells plated on damaged matrix $(P<0.05)$, but this increase was reduced by exogenous BDNF treatment $(P<0.05)$ (Figure $5 \mathrm{G}$ ). This supports a role for BDNF both upstream of YAP/WWTR1 in calponin expression and downstream of YAP/WWTR1 in myosin expression.

BDNF Induces a Loss of Normal Contractile Force That Depends on BDNF Receptor Tyrosine Kinase Activity

Pretreatment of cultures of bladder smooth muscle cells with exogenous BDNF compared with vehicle was able to prevent normal levels of smooth muscle cell contraction in collagen gel contraction assays after 4 and 18 hours (by $14 \%$ and $23 \%$, respectively). Interestingly, GNF 5837, an inhibitor of BDNF signaling through neurotrophic receptor
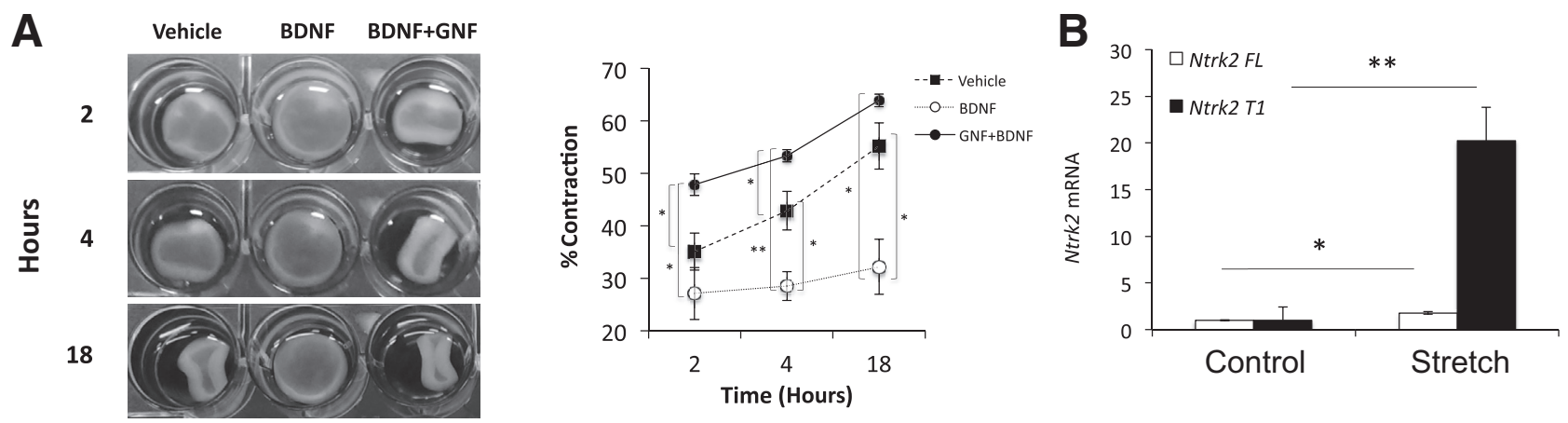

Figure 6 Smooth muscle contraction is inhibited by brain-derived neurotrophic factor (BDNF) treatment and reversed by neurotrophic receptor tyrosine kinase (NTRK)2 kinase inhibition. A: Human bladder smooth muscle cells (BSMCs) plated on collagen gels induce contraction, but it is inhibited by pretreatment of cells with $10 \mathrm{ng} / \mathrm{mL}$ BDNF. Addition of $10 \mu \mathrm{mol} / \mathrm{L}$ GNF 5837, an inhibitor of NTRK2 kinase activity, reverses the relaxation response of BDNF and enhances the contractile response significantly. B: To examine Ntrk2 expression levels during mechanical stretch, real-time quantitative PCR was performed on rat BSMCs that were mechanically stretched using a slowly ramping pattern to $5 \%$ elongation at 6 hours. Mechanical strain of rat bladder smooth muscle cells augments mRNA expression of Ntrk2 full-length and truncated isoform. ${ }^{\star} P<0.05,{ }^{*} P<0.01$. 
tyrosine kinase (NTRK)2, was able to reverse the lack of contraction, increasing the BDNF contractile response by $20.7 \%, 24.8 \%$, and $31.8 \%$ at 2,4 , and 18 hours, respectively $(P<0.005)$ (Figure 6A). The GNF $5837+$ BDNF-induced contraction was even more pronounced than vehicle, increasing $12.7 \%$ and $10.5 \%$ at 2 and 4 hours, respectively. Real-time quantitative PCR was performed to examine if Ntrk2 was altered during obstruction in vivo. Ntrk2 expression of neither the total nor the truncated (T1) isoform in bladder tissues was altered significantly during obstruction or DAC treatment (data not shown). Because the expression from whole bladder is a mix of cell lineages, Ntrk2 isoforms were examined in rat bladder smooth muscle cells. Interestingly, total and T1 Ntrk2 increased 1.8 -fold $(P<0.05)$ and 20.2 -fold $(P<0.005)$ during stretch versus no-stretch BSMCs (Figure 6B).

\section{Discussion}

In this study, pharmacologic inhibition (induced loss) of DNA methylation leads to frequent and inefficient voiding, as well as marked dilative and hypertrophic changes in the obstructed bladder. Our findings suggest that DNA methylation is crucial for regulation of bladder pathophysiology incited by PBO. Bladder myopathies characterized by obstruction and loss of controlled storage and emptying functions increase in aging populations who are prone to a global loss of DNA methylation. ${ }^{16,17}$ HT-qPCR of candidate genes revealed that Bdnf, Cyr61, and Ctgf comprised a small cluster of genes up-regulated by obstruction. Moreover, this obstruction-induced cluster was further amplified in the presence of DAC. Bdnf expression demonstrated a quantitative link to several pathophysiologic changes relating to growth, SMC hypertrophy, and loss of voiding efficiency. $B d n f$ was initially selected as a relevant candidate gene in bladder pathophysiology because of its upregulation during obstruction and implied association with the pathomechanics of obstruction. More specifically, it was found that Bdnf and Ctgf associate with physiological changes during obstruction and their expression is mechanically induced, which is dependent on YAP/WWTR1 signaling.

\section{BDNF Is Regulated by DNA Methylation, WWTR1, and} YAP

Regulation of BDNF expression relies heavily on $\mathrm{CpG}$ demethylation and methylation in concert with chromatin and transcription factor binding. ${ }^{10,15}$ However, $B D N F$ promoter CpG DNA methylation in our samples did not reveal large changes in $\mathrm{CpG}$ methylation of the promoter region. The largely stable DNA methylation pattern in $B D N F$ so far uncovered contrasts with the dynamically regulated in vitro BSMC DNA methylome. In a previous study, an induction of genome-wide methylation changes was observed in BSMCs after exposure to denatured extracellular matrix for only 2 days. $^{3}$ Interestingly, there was a global hypomethylation of the BSMC DNA alongside localized hypermethylation. ${ }^{3}$ More important, this may relate to the known plasticity of SMCs. Mature normal SMCs demonstrate a high level of hydroxy-methyl-cytosine (HO$\mathrm{meCpG}),{ }^{18,19}$ similar to that of stem cells. SMCs have been suggested to use $\mathrm{HO}$ methylation as a mechanism to respond to highly changeable environments. ${ }^{18,19}$

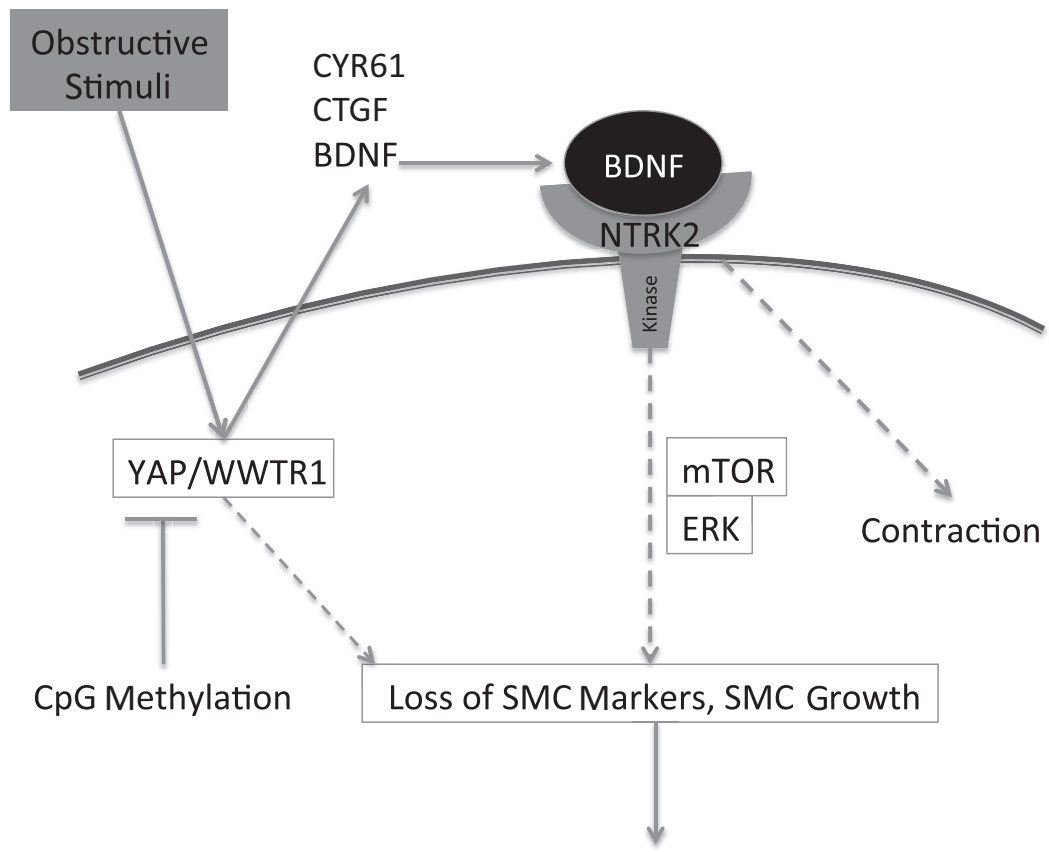

Figure 7 Schematic pathway describes the dependency of smooth muscle cell (SMC) dedifferentiation induced by obstructive bladder stimuli, such as strain on YAP/WWTR1 to produce brain-derived neurotrophic factor (BDNF), alongside CTGF and CYR61. Inhibition of DNA methylation leads to increased YAP and WWTR1, which leads to increased BDNF and CTGF. BDNF itself leads to increased SMC growth and loss of some myosin expression, in a time- and dose-dependent manner. Growth pathways, which have been linked to bladder SMC growth, are activated by BDNF. Inhibition of YAP/WWTR1 increases expression of SMC markers that are downregulated by BDNF, suggesting some combined effects of YAP/WWTR1 and BDNF. Furthermore, blocking either BDNF receptors or YAP/WWTR1 leads to increase in SMC marker expression. ERK, extracellular signal-regulated kinase; mTOR, mammalian target of rapamycin. 
Interestingly, decitabine can increase $\mathrm{HO}-\mathrm{meCpG}$ levels in leukemic cells, Jurkat cells, and neural stem cells, ${ }^{20,21}$ but little is known about HO-mCpG during decitabine treatment of SMCs in obstruction. ${ }^{22}$ One focus of our future work centers on $\mathrm{HO}-\mathrm{mCpG}$ and $\mathrm{mCpG}$ alterations of transcription factor binding in promoter regions of BDNF, CYR61, or $C T G F$, and the potential instability of $\mathrm{HO}$ methylation in $\mathrm{PBO}$ as a stepping stone to demethylation.

$\mathrm{CpG}$ methylation changes in transcriptional regulators may up-regulate expression of BDNF and CYR61 + CTGF. To search for this mechanism of increased BDNF, CYR61, and CTGF, pathway analysis using TOPGO and OPOSSUM unveiled a common YAP/WWTR1 and TEAD binding element among the coregulated genes. DAC treatment greatly increases transcription of WWTR1 in vitro in conjunction with downstream targets, CYR61 + CTGF and BDNF. Protein levels of BDNF by enzymelinked immunosorbent assay also increased during obstruction plus treatment, but not as much as expected given the mRNA levels. This may be because of increased antisense RNA against Bdnf mRNA, which regulates transcript stability. Other transcriptional mechanisms altering $B d n f$ mRNA may include MECP2, which is inhibitory in general. MECP2's potential role in disinhibition of BDNF during obstruction with or without DAC is not consistent, because Mecp2 mRNA levels increase during obstruction when $B d n f$ mRNA is increasing, yet decrease with DAC treatment during obstruction (Supplemental Figure S9). More important, WWTR1 mRNA levels themselves were altered by DAC treatment both in vivo and in vitro (Figures $3 \mathbf{J}$ and $4 \mathrm{~A}$ ), suggesting that the increase in the Bdnf and Cyr61 + Ctgf may be related to the increased expression of their transcriptional cofactors. Interestingly, WWTR1 and YAP staining indicated that there is likely some regulation at the protein level, because a significant amount of protein was seen in the cytoplasm of the PBO + vehicle and sham + DAC groups. Because many transcription factors interact with WWTR1 and YAP to increase their effects downstream, TEAD expression, which is associated with YAP/WWTR1 activity, was examined. However, the TEADs detected are not significantly altered by obstruction or DAC. YAP and WWTR1 are also regulated post-translationally, which was addressed through an examination of YAP/WWTR1 expression and nuclear localization (Figures 3 and 4D). In vivo, Yap antibody staining showed that phosphorylated Yap, which is most often shunted for degradation, appeared to be reduced during obstruction. This was concurrent with increased nuclear localization in the obstructed groups, with the decitabine treatment increasing localization further. The implication is that DNA methylation normally restricts WWTR1 and YAP expression and localization patterns, but unrestricted YAP and WWTR1 expression leads to vastly up-regulated CTGF and BDNF, damaging the bladder. YAP and WWTR1 are coactivators, which associate with DNA only through the DNA binding activity of other transcription factors, such as early growth reponse 1 (EGR1), T-box 5, $\beta$-catenin, and myocardin. Critically, YAP and WWTR1 are involved in the regulation of BDNF during mechanical stimulation through binding to its promoter. An EGR1 binding site in the $B D N F$ promoter is one target for mechanosensitive binding of YAP/WWTR1 (Figure 3I). EGR1 is a transcription factor, which binds DNA and YAP during proliferation, mechanical stretch, ${ }^{23}$ and inflammation. ${ }^{24,25}$ In summary, DNA methylation, in part, regulates BDNF through the regulation of the YAP and WWTR1 transcription and localization.

\section{BDNF Regulates Smooth Muscle Differentiation}

Although many studies have examined BDNF in terms of its effects on neuronal function in the bladder, ${ }^{4,5,26-28}$ few have examined its direct role in the BSMC phenotype. The myogenic hypothesis in obstructive and overactive bladder is supported by work examining direct effects of cytokines and other factors on the detrusor phenotype and function. ${ }^{29-31}$ However, BDNF alters survival, proliferation, synthetic activity, and contractile functions of many cell types from many organs. BDNF's role in lung and vascular SMC differentiation, contraction, and proliferation has been explored in a few limited studies. Together, this suggests that a more direct role for BDNF should be considered in the function of bladder smooth muscle than previously suggested, ${ }^{5}$ especially given the elevated level of BDNF in the detrusor. Interestingly, basal BDNF in the bladder exceeds basal expression of all other organs, even the brain. ${ }^{15}$ Furthermore, the PBO+DAC level of BDNF expression is 100 -fold higher than in sham bladder. This high level of bladder BDNF could have widespread effects on neuronal and nonneuronal cell types. The high levels of BDNF immediately surrounding the smooth muscle bundles in the endomysial spaces in this study (Figure 7), during obstruction, suggest that BDNF may target muscle bundles and their surrounding cells. Further supporting a direct role of BDNF on SMC function, it was found that BDNF was able to alter markers of smooth muscle differentiation (eg, its smooth muscle myosin heavy chain and calponin expression), which was consistent with the loss of differentiation in the obstructed bladders.

\section{BDNF Signals to Proproliferative Pathways in Bladder Smooth Muscle}

BDNF can signal through the mTOR pathway, such as S6 kinase (S6K), elongation initiation factor 4E, mitogenactivated protein kinase-1, and mitogen-activated protein kinase- $3,{ }^{32}$ which are also activated in both the urothelium and mesenchyme during SMC strain and bladder obstruction. ${ }^{6,10,11}$ Previously, it was found that mTOR pathway is activated during $\mathrm{PBO},{ }^{6}$ and induces smooth muscle hypertrophy during PBO. SMC dedifferentiation is regulated by 
S6K1 in vitro. ${ }^{11,33}$ Conversely vascular SMC differentiation is improved by inhibition of mTOR complex 1 through a combination of blocking S6K1, insulin receptor substrate$1 / 2$, and GATA binding protein $6 .{ }^{11,33}$ However, inhibition of S6K as opposed to the entire mTOR pathway in mice during obstruction lead to worsening of bladder hypertrophy. ${ }^{7}$ Herein, it was observed that BDNF induces extracellular signal-regulated kinase and S6K1 and is associated with loss of differentiation in BSMCs, consistent with previous studies. ${ }^{10}$ In particular, high-dose sustained BDNF signaling may play a role in bladders by causing sustained extracellular signal-regulated kinase signaling, which is known to have differential effects on BSMC growth and neurons. ${ }^{10}$ Although the in vivo role of BDNF signaling in smooth muscle cells remains to be further elucidated, it is clear that DNA methylation is playing a role in BDNF regulation in vivo and in vitro.

Other pathways may also be regulating SMC marker genes and myocardin during decitabine treatment, including serum response factor. ${ }^{34}$ In supplementary HTqPCR data (Supplemental Figure S8), serum response factor was down-regulated by DAC treatment, showing the opposite pattern of expression to BDNF during obstruction and DAC treatment. Serum response factor increased nonsignificantly during obstruction, but decreased with DAC. MECP2 is also known to have a role in BDNF regulation, ${ }^{35}$ but as mentioned above, MECP2 expression is not concordant with BDNF expression in vivo (Supplemental Figure S9).

\section{YAP and WWTR1 Inhibition Improves Smooth Muscle Phenotype by Down-Regulating BDNF}

For the first time, we observe that YAP/WWTR1 regulation of BDNF by mechanosensitive stimuli may be a crucial mechanism that leads to aberrant gene regulation of a subset of genes and discrete alterations in BSMC phenotype and function. As such, this is the first report to show that YAP and WWTR1 play a central role in the mechanoregulation of BDNF, CYR61, and CTGF in bladder smooth muscle. In addition, BDNF's effects were dose dependent, which may have an impact on the varying effects of BDNF in vivo. In some reports, BDNF has been suggested as a possible therapy, ${ }^{5}$ whereas in others, its association with hyperactivity is described. ${ }^{36}$ The diverging results may relate to dosage of BDNF. Dose-dependent effects of BDNF have been noted in other cell types. ${ }^{32}$ In addition, locally expressed versus systemically introduced BDNF may have diverging effects on bladder pathophysiology. Systemic BDNF may be beneficial for neuronal control of the bladder, through improved neuronal sprouting, neuronal survival, cognition, recovery from neuronal trauma, and prevention of social stress, but these effects might not be beneficial in the local bladder setting. Local high levels of BDNF likely induce growth and loss of smooth muscle cell markers, as in vitro (Figure 5), which may affect the BDNF-correlated pathophysiology, including micturition, residual volume, and hypertrophy.

\section{BDNF Alters Contractile Function of Bladder Smooth Muscle through Nonkinase Activity of NTRK2}

The loss of contractile function in response to BDNF was consistent with the effects of BDNF on differentiation of SMCs. It is known that BDNF can increase contractile frequency of bladder strips and the bladder in vivo, but the effect of BDNF on bladder smooth muscle phenotype shown herein provides new information that may support a role for loss of micturition efficiency (Figure 1). Interestingly, the nerve density (Supplemental Figure S11) coincided within the BDNF elevation during $\mathrm{PBO}+\mathrm{DAC}$ versus $\mathrm{PBO}$ alone, although not during $\mathrm{PBO}$ versus sham. However, the role of the main BDNF receptor, NTRK2, has divergent effects on gel contraction (Figure 6A), as the isoforms of the receptor determine its signaling capacity (Figure 7). Inhibition of the tyrosine kinase of NTRK2 with GNF 5837 promotes gel contraction. This is consistent with the known activity of the partially truncated NTRK2 isoform T1, which lacks the kinase activity but retains $\mathrm{Ca}^{+2}$ - and Rho kinase-mediated contractile signaling. Our results suggest that gel contraction is promoted by the nonkinase isoform of NTRK2 T1. However, other pathways (eg, muscarinic and adrenergic) participate in bladder smooth muscle contraction in vivo, although these ultimately feed into sarcoplasmic reticulum regulation of calcium. Furthermore, relaxation of BSMCs has been studied mainly as a consequence of inhibition of contraction, but in the heart, BDNF signaling through phospholipase $\mathrm{C}$ appears to have an important role in ensuring the relaxation phase. ${ }^{37}$ Silencing the $\mathrm{T} 1$ isoform in the presence of BDNF overexpression is associated with reduced Rho kinase signaling and decreased activity, whereas BDNF overexpression can alter neuronal density and activity, leading to bladder hyperactivity. Although the mRNA of Ntrk2 full-length and truncated isoforms was increased in vitro by stretch and NTRK was found to play a role in regulating relaxation and contraction in vitro, Ntrk2 effects in vivo were not in the scope of this article, and form the focus of future studies. This study demonstrates the association of SMCs and gross bladder hypertrophy with BDNF as a downstream target of YAP and WWTR1.

\section{Conclusions}

Pathophysiologic parameters of obstruction are associated with expression of $B d n f$, concordant with a direct role for BDNF in control of SMC phenotype. DNA methylation inhibition augments $B d n f$ expression in BSMCs in vitro and during partial obstruction of the bladder in vivo, suggesting $B d n f$ is normally repressed by DNA methylation. 
Furthermore, Ctgf and Bdnf expression are modulated by WWTR1 and YAP, which in turn are epigenetically regulated by DNA methylation in vitro and in vivo.

\section{Acknowledgment}

We thank the SickKids Imaging Facility and Lab Animal Services for technical assistance.

\section{Supplemental Data}

Supplemental material for this article can be found at https://doi.org/10.1016/j.ajpath.2018.06.024.

\section{References}

1. Ganz ML, Smalarz AM, Krupski TL, Anger JT, Hu JC, WittrupJensen KU, Pashos CL: Economic costs of overactive bladder in the United States. Urology 2010, 75:526-532.e18

2. Tao H, Yang J-J, Shi K-H, Deng Z-Y, Li J: DNA methylation in cardiac fibrosis: new advances and perspectives. Toxicology 2014, 323:125-129

3. Jiang J-X, Aitken KJ, Sotiropoulos C, Sotiropolous C, Kirwan T, Panchal T, Zhang N, Pu S, Wodak S, Tolg C, Bägli DJ: Phenotypic switching induced by damaged matrix is associated with DNA methyltransferase 3A (DNMT3A) activity and nuclear localization in smooth muscle cells (SMC). PLoS One 2013, 8:e69089

4. Cruz CD: Neurotrophins in bladder function: what do we know and where do we go from here? Neurourol Urodyn 2014, 33:39-45

5. Song Q-X, Chermansky CJ, Birder LA, Li L, Damaser MS: Brainderived neurotrophic factor in urinary continence and incontinence. Nat Rev Urol 2014, 11:579-588

6. Schröder A, Kirwan TP, Jiang J-X, Aitken KJ, Bägli DJ: Rapamycin attenuates bladder hypertrophy during long-term outlet obstruction in vivo: tissue, matrix and mechanistic insights. J Urol 2013, 189: 2377-2384

7. Sidler M, Aitken KJ, Forward S, Vitkin A, Bägli DJ: Non-invasive voiding assessment in conscious mice. Bladder 2018, 5:e33

8. Sidler M, Aitken K, Jiang J, Bijos D, Belik J, Bägli DJ: Finding NeMO-nerve-sparing mid-urethral obstruction: a pathophysiologically accurate model of rodent partial bladder outlet obstruction. Urology 2017, 105:208.e1-208.e9

9. Sidler M, Aitken KJ, Jiang J-X, Bägli DJ: Nerve-sparing mid-urethral obstruction (NeMO) in female small rodents. J Vis Exp 2017, 122: e55288

10. Chen KW, Chen L: Epigenetic regulation of BDNF gene during development and diseases. Int J Mol Sci 2017, 18:pii:E571

11. Aitken KJ, Tolg C, Panchal T, Leslie B, Yu J, Elkelini M, Sabha N, Tse DJ, Lorenzo AJ, Hassouna M, Bägli DJ: Mammalian target of rapamycin (mTOR) induces proliferation and de-differentiation responses to three coordinate pathophysiologic stimuli (mechanical strain, hypoxia, and extracellular matrix remodeling) in rat bladder smooth muscle. Am J Pathol 2010, 176:304-319

12. Herz DB, Aitken K, Bägli DJ: Collagen directly stimulates bladder smooth muscle cell growth in vitro: regulation by extracellular regulated mitogen activated protein kinase. J Urol 2003, 170: 2072-2076

13. Dvinge H, Bertone P: HTqPCR: high-throughput analysis and visualization of quantitative real-time PCR data in R. Bioinformatics 2009, 25:3325-3326

14. Tolg C, Ahsan A, Dworski S, Kirwan T, Yu J, Aitken K, Bägli DJ: Pathologic bladder microenvironment attenuates smooth muscle differentiation of skin derived precursor cells: implications for tissue regeneration. PLoS One 2013, 8:e59413

15. Lommatzsch M1, Braun A, Mannsfeldt A, Botchkarev VA, Botchkareva NV, Paus R, Fischer A, Lewin GR, Renz H: Abundant production of brain-derived neurotrophic factor by adult visceral epithelia. Implications for paracrine and target-derived neurotrophic functions. Am J Pathol 1999, 155:1183-1193

16. Wilson AS, Power BE, Molloy PL: DNA hypomethylation and human diseases. Biochim Biophys Acta 2007, 1775:138-162

17. Richardson B: Impact of aging on DNA methylation. Ageing Res Rev 2003, 2:245-261

18. Liu R, Leslie KL, Martin KA: Epigenetic regulation of smooth muscle cell plasticity. Biochim Biophys Acta 2015, 1849:448-453

19. Liu R, Jin Y, Tang WH, Qin L, Zhang X, Tellides G, Hwa J, Yu J, Martin KA: Ten-eleven translocation-2 (TET2) is a master regulator of smooth muscle cell plasticity. Circulation 2013, 128:2047-2057

20. Chowdhury B, McGovern A, Cui Y, Choudhury SR, Cho I-H, Cooper B, Chevassut $\mathrm{T}$, Lossie AC, Irudayaraj $\mathrm{J}$ : The hypomethylating agent decitabine causes a paradoxical increase in 5hydroxymethylcytosine in human leukemia cells. Sci Rep 2015, 5: 9281

21. Karagiannis TC, Maulik N: Factors influencing epigenetic mechanisms and related diseases. Antioxid Redox Signal 2012, 17:192-194

22. Zhuang J, Luan P, Li H, Wang K, Zhang P, Xu Y, Peng W: The Yin-Yang dynamics of DNA methylation is the key regulator for smooth muscle cell phenotype switch and vascular remodeling. Arterioscler Thromb Vasc Biol 2017, 37:84-97

23. Pardo PS, Mohamed JS, Lopez MA, Boriek AM: Induction of Sirt1 by mechanical stretch of skeletal muscle through the early response factor EGR1 triggers an antioxidative response. J Biol Chem 2011, 286: 2559-2566

24. Tureyen K, Brooks N, Bowen K, Svaren J, Vemuganti R: Transcription factor early growth response-1 induction mediates inflammatory gene expression and brain damage following transient focal ischemia. J Neurochem 2008, 105:1313-1324

25. Zagurovskaya M, Shareef MM, Das A, Reeves A: EGR-1 forms a complex with YAP-1 and upregulates Bax expression in irradiated prostate carcinoma cells. Oncogene 2009, 28:1121-1131

26. Ochodnicky P, Cruz CD, Yoshimura N, Cruz F: Neurotrophins as regulators of urinary bladder function. Nat Rev Urol 2012, 9: 628-637

27. Antunes-Lopes T, Pinto R, Barros SC, Botelho F, Silva CM, Cruz CD, Cruz F: Urinary neurotrophic factors in healthy individuals and patients with overactive bladder. J Urol 2013, 189:359-365

28. Yuk SM, Shin JH, Song KH, Na YG, Lim JS, Sul CK: Expression of brain derived-neurotrophic factor and granulocyte-colony stimulating factor in the urothelium: relation with voiding function. BMC Urol $2015,15: 37$

29. Hashemi Gheinani A, Burkhard FC, Rehrauer H, Aquino Fournier C, Monastyrskaya K: MicroRNA MiR-199a-5p regulates smooth muscle cell proliferation and morphology by targeting WNT2 signaling pathway. J Biol Chem 2015, 290:7067-7086

30. Imamura M, Kanematsu A, Yamamoto S, Kimura Y, Kanatani I, Ito N, Tabata Y, Ogawa O: Basic fibroblast growth factor modulates proliferation and collagen expression in urinary bladder smooth muscle cells. Am J Physiol Renal Physiol 2007, 293:F1007-F1017

31. Metcalfe PD, Wang J, Jiao H, Huang Y, Hori K, Moore RB, Tredget EE: Bladder outlet obstruction: progression from inflammation to fibrosis. BJU Int 2010, 106:1686-1694

32. Santos D, Gonzalez-Perez F, Navarro X, Del Valle J: Dose-dependent differential effect of neurotrophic factors on in vitro and in vivo regeneration of motor and sensory neurons. Neural Plast 2016, 2016: 4969523

33. Martin KA, Rzucidlo EM, Merenick BL, Fingar DC, Brown DJ, Wagner RJ, Powell RJ: The mTOR/p70 S6K1 pathway regulates vascular smooth muscle cell differentiation. Am J Physiol Cell Physiol 2004, 286:C507-C517 
34. Wang Z1, Wang DZ, Hockemeyer D, McAnally J, Nordheim A, Olson EN: Myocardin and ternary complex factors compete for SRF to control smooth muscle gene expression. Nature 2004, 428:185-189

35. Sampathkumar C, Wu Y-J, Vadhvani M, Trimbuch T, Eickholt B, Rosenmund C: Loss of MeCP2 disrupts cell autonomous and autocrine BDNF signaling in mouse glutamatergic neurons. Elife 2016, 5:214

36. Frias B, Santos J, Morgado M, Sousa MM, Gray SMY, McCloskey KD, Allen S, Cruz F, Cruz CD: The role of brain- derived neurotrophic factor (BDNF) in the development of neurogenic detrusor overactivity (NDO). J Neurosci 2015, 35: 2146-2160

37. Feng N, Huke S, Zhu G, Tocchetti CG, Shi S, Aiba T, Kaludercic N, Hoover DB, Beck SE, Mankowski JL, Tomaselli GF, Bers DM, Kass DA, Paolocci N: Constitutive BDNF/TrkB signaling is required for normal cardiac contraction and relaxation. Proc Natl Acad Sci U S A $2015,112: 1880-1885$ 